\title{
LATTICE-ORDERED ABELIAN GROUPS AND SCHAUDER BASES OF UNIMODULAR FANS, II
}

\author{
VINCENZO MARRA
}

Dedicated to A.M.W. Glass on the occasion of his retirement.

\begin{abstract}
Unimodular fans are central to toric algebraic geometry, where they correspond to non-singular toric varieties. The Schauder bases mentioned in the title may be described as the standard bases of the free $\mathbb{Z}$-module of support functions (=invariant Cartier divisors) of a unimodular (a.k.a. regular) fan. An abstract, purely algebraic version of Schauder bases was investigated in the first part of the present paper, with motivations coming from the theory of lattice-ordered Abelian groups. The main result obtained there is that such abstract Schauder bases can be characterised in terms of the maximal spectral space of lattice-ordered Abelian groups, with no reference to polyhedral geometry. The results in the present paper will show that abstract Schauder bases can in fact be characterised in the language of lattice-ordered groups by means of an elementary algebraic notion that we call regularity, with no reference to either maximal spectral spaces or to polyhedral geometry. We prove that finitely generated projective lattice-ordered Abelian groups are precisely the lattice-ordered Abelian groups that have a finite, regular set of positive generators. This theorem complements Beynon's well-known 1977 result that the finitely generated projective lattice-ordered Abelian groups are precisely the finitely presented ones; and the core of the proof consists in showing that finite, regular sets of positive generators are the same thing as abstract Schauder bases. We give three applications of the main result. First, we establish a necessary and sufficient criterion for the lattice-group isomorphism of two lattice-ordered Abelian groups with finite, regular sets of positive generators. Next, we classify in elementary terms (i.e. without reference to spectral spaces) all finitely generated projective lattice-ordered Abelian groups whose maximal spectrum is a closed topological surface. Finally, we show how to explicitly construct $\mathbb{Z}$-module bases of any finitely generated projective lattice-ordered Abelian group.
\end{abstract}

\section{INTRODUCTION}

Unimodular fans 1 are central to toric algebraic geometry (see e.g. [27]), where they correspond to non-singular toric varieties. Schauder bases [29, 24] over a unimodular fan $\Delta$ in $\mathbb{R}^{n}$ may be regarded as the standard bases of the free $\mathbb{Z}$ module SF $(\Delta)$ of support functions of the fan, in the sense of [27, Ch. 2]. Their significance in toric geometry is due to the fact that $\operatorname{SF}(\Delta)$ is isomorphic to the

Received by the editors July 13, 2011 and, in revised form, September 8, 2011.

2010 Mathematics Subject Classification. Primary 06F20, 52B20, 08B30; Secondary 06B25, $55 \mathrm{~N} 10$.

Key words and phrases. Lattice-ordered Abelian group, free $\ell$-group, projective $\ell$-group, unimodular fan, regular fan, maximal spectral space, pairwise disjointness, linear independence, Euler characteristic, $\mathbb{Z}$-module basis.

${ }^{1}$ Unimodular fans are also called regular or non-singular in the literature on toric varieties. Here we follow the terminology of [20], for the sake of consistency. 
group of torus-invariant Cartier divisors on the toric variety associated to $\Delta$; see [27, §2.1]. The group $\mathrm{SF}(\Delta)$ sits inside the much larger group of all real-valued piecewise homogenous linear functions on the underlying polyhedron of $\Delta$ that have linear pieces with integer coefficients. When ordered pointwise, this group is an instance of a lattice-ordered group [7, 11, 14, or $\ell$-group for short - that is, a group that is also a lattice, and is such that the group operation distributes over both lattice meets and joins. The order that $\mathrm{SF}(\Delta)$ inherits from this $\ell$-group has a natural algebro-geometric meaning, too: the non-negative support functions correspond precisely to the effective torus-invariant Cartier divisors (=Weil divisors, because of non-singularity) on the toric variety associated to $\Delta$ [27, pp. 68-69].

Abstract Schauder bases were defined and investigated in the first part [20], with motivations coming from the theory of $\ell$-groups. An abstract Schauder basis of an Abelian $\ell$-group is defined as a subset of elements that admits a representation as the Schauder basis over some unimodular fan, up to an isomorphism of $\ell$-groups; see Subsection 2.1 for precise definitions. The main result of [20] is that such subsets of elements can be intrinsically characterised in terms of the maximal spectral space of the $\ell$-group, with no reference to polyhedral geometry. The results in the present paper will show that abstract Schauder bases can in fact be characterised in the language of lattice-ordered groups via an elementary algebraic definition, with no reference to either maximal spectral spaces or to representations of $\ell$-groups by piecewise linear functions.

We henceforth assume familiarity with lattice-ordered groups. Since all $\ell$-groups in this paper are Abelian, we often write " $\ell$-group" to mean "Abelian $\ell$-group". We write $\ell$-groups additively, with neutral element 0 ; the lattice meet (join) is written $\wedge(\vee)$. By $\ell$-homomorphisms we mean homomorphisms of $\ell$-groups, i.e. latticegroup homomorphisms; the symbol " $\cong$ " denotes the existence of an $\ell$-isomorphism, whereas $\cong$ is reserved for group isomorphism. Throughout the paper, $\mathbb{Z}$ is the set of integers, $\mathbb{R}$ is the set of real numbers, "positive" means "strictly positive", and therefore "non-negative" means "positive or zero". For any $\ell$-group $G$, we set $G^{+}=\{g \in G \mid g \geqslant 0\}$. For background on polyhedra, simplicial complexes, and polyhedral fans, we refer to 28 and 13 .

Abelian $\ell$-groups form a finitary algebraic category (a variety of algebras, in alternative terminology). Therefore, free Abelian $\ell$-groups exist over free generating sets of any cardinality. Beynon proved [6, Thm. 3.1] (cf. also [3, Thm. 5.1]) that $\ell$-groups arising from unimodular fans in the fashion above are precisely those that are finitely generated and projective; and that they also coincide with finitely presented $\ell$-groups, that is, with quotients of free finitely generated $\ell$-groups modulo a finitely generated congruence. Here, an $\ell$-group $P$ is projective if for any onto $\ell$-homomorphism $e: X \rightarrow Y$, and $\ell$-homomorphism $f: P \rightarrow Y$, there is an $\ell$ homomorphism $g: P \rightarrow X$ such that $f=e \circ g$, i.e. such that the following diagram commutes.

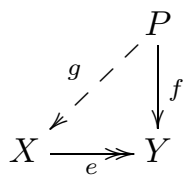

Free $\ell$-groups are projective [10, 3.8.6], and $P$ is projective if and only if it is a retract of a free object [9, 4.6.4]; that is, if there is an $\ell$-homomorphism $r: F \rightarrow P$, with $F$ a free $\ell$-group, such that there exists an $\ell$-homomorphism $s: P \rightarrow F$ with 
$r \circ s$ the identity on $P$. When that is the case, the retraction $r$ must be onto, and the section $s$ must be into. These facts have nothing to do with $\ell$-groups in particular: they hold, mutatis mutandis, in any algebraic category. By contrast, Beynon's above-mentioned theorem is quite specific to the theory of $\ell$-groups. Our main result is an alternative, completely elementary characterisation of finitely generated projective $\ell$-groups.

Given a finite set $B=\left\{b_{1}, \ldots, b_{t}\right\}$ of positive elements of an $\ell$-group $G$, we say $B$ is starrable at $\left\{b_{r}, b_{s}\right\} \subseteq B$, where $r<s$, if $b_{r} \wedge b_{s} \neq 0$. In that case, the stellar subdivision of $B$ at $\left\{b_{r}, b_{s}\right\}$ is the set

$$
B_{b_{r}, b_{s}}=\left\{b_{1}^{\prime}, \ldots, b_{t}^{\prime}, b_{t+1}^{\prime}\right\} \backslash\{0\},
$$

where $\backslash$ denotes set-theoretic difference, and

$$
\begin{aligned}
b_{r}^{\prime} & =b_{r}-\left(b_{r} \wedge b_{s}\right), \\
b_{s}^{\prime} & =b_{s}-\left(b_{r} \wedge b_{s}\right), \\
b_{i}^{\prime} & =b_{i} \text { for } 1 \leqslant i \leqslant t, r \neq i \neq s, \\
b_{t+1}^{\prime} & =b_{r} \wedge b_{s} .
\end{aligned}
$$

We say that $B_{b_{r}, b_{s}}$ is obtained from $B$ via a stellar subdivision (at $\left\{b_{r}, b_{s}\right\}$ ). Cf. [20. Lemma 2.4 and Prop. 5.2]; see also [21, Prop. 2.2 and proof of Lemma 4.3] for an early application of stellar subdivisions, in the sense above, to $\ell$-groups.

Definition. Let $G$ be a lattice-ordered Abelian group, and let $B=\left\{b_{1}, \ldots, b_{t}\right\} \subseteq$ $G^{+} \backslash\{0\}$ for some integer $t \geqslant 0$.

(R1) $B$ is 1-regular if whenever $B$ is starrable at $\left\{b_{r}, b_{s}\right\} \subseteq B$, then, with $B_{b_{r}, b_{s}}$ as in (*), the following hold. For any $1 \leqslant i_{1}<\cdots<i_{k} \leqslant t$, if

$$
\left(b_{r} \wedge b_{s}\right) \wedge b_{i_{1}} \wedge \cdots \wedge b_{i_{k}}>0 \text { holds in } G
$$

then for every $\emptyset \neq J \subseteq\left\{i_{1}, \ldots, i_{k}\right\}$ with $\{r, s\} \nsubseteq J$

$$
\left(b_{r} \wedge b_{s}\right) \wedge \bigwedge_{j \in J} b_{j}^{\prime}>0 \text { holds in } G .
$$

(R2) $B$ is regular if it is 1-regular, and whenever $B$ is starrable at $\left\{b_{r}, b_{s}\right\} \subseteq B$, then $B_{b_{r}, b_{s}}$ is again 1-regular.

Regularity generalises both pairwise disjointness (in the lattice $G^{+}$with bottom element 0 ) and linear independence (in the $\mathbb{Z}$-module $G$ ) of sets of positive elements. For if $B$ as in the above is a set of pairwise disjoint elements (meaning $h \wedge k=0$ for every $h \neq k \in B$ ), then $B$ is trivially regular, because it is starrable at no pair $\{h, k\} \subseteq B$. It is considerably harder to show, but nonetheless true, that if $B$ is linearly dependent in $G$, then $B$ cannot be regular; see the Remark after the proof of Lemma 2.6 below. To illustrate further: if $G$ is totally ordered, and if the regular set $\emptyset \neq B \subseteq G^{+} \backslash\{0\}$ generates $G$, then $G$ is $\mathbb{Z}$ and $B$ is $\{1\}$, up to an $\ell$-isomorphism. Indeed, if we had $|B|>1$, then $B$ would be starrable at each pair $\{h, k\} \subseteq B-$ because $G$ is totally ordered - and $B$ would not be 1-regular. To see this, say $h>k$ holds. Then $k-(h \wedge k)=0$, and thus $(h \wedge k) \wedge(k-(h \wedge k))=0$ whereas $(h \wedge k) \wedge k=k>0$, contradicting (R1). Therefore $|B|=1$, and $G$ is totally ordered singly generated; the conclusion follows. In this example, observe that $G \cong{ }_{\ell} \mathbb{Z}$ is projective, because $\mathbb{Z}^{2}$ with pointwise order is known to be the free $\ell$-group generated by $(1,-1)$, and the unique onto $\ell$-homomorphism $\mathbb{Z}^{2} \rightarrow \mathbb{Z}$ that extends 
the function $(1,-1) \in \mathbb{Z}^{2} \longmapsto 1 \in \mathbb{Z}$ admits the section $z \in \mathbb{Z} \longmapsto(1,0) \in \mathbb{Z}^{2}$. Far more generally:

Theorem. For any lattice-ordered Abelian group $G$, the following are equivalent:

(1) $G$ is finitely generated and projective.

(2) $G$ has a finite, regular set of positive generators.

This theorem is the main result of the present paper; it is proved in Section 2 .

We now state a corollary which holds independent interest. Let $B$ be a finite set of positive elements of an $\ell$-group $G$. With $B$, let us associate the set $\mathscr{S}(B)$ of subsets of $B$ such that $\emptyset \in \mathscr{S}(B)$, and $\emptyset \neq B^{\prime} \subseteq B$ lies in $\mathscr{S}(B)$ if and only if $\bigwedge B^{\prime} \neq 0$ in $G$. (Cf. [20, p. 1596].) Then $B^{\prime \prime} \subseteq B^{\prime} \in \mathscr{S}(B)$ implies $B^{\prime \prime} \in \mathscr{S}(B)$, so that $\mathscr{S}(B)$ is an abstract simplicial complex, i.e. a family of subsets of $B$ that is closed under the operation of taking subsets, and includes all singletons. The subsets of $\mathscr{S}(B)$ of cardinality $k+1$ are called $k$-dimensional (abstract) simplices, or $k$-simplices for short; the dimension of $\mathscr{S}(B)$ is the maximum of the dimensions of its simplices. Singleton simplices are also called vertices of the complex. The simplicial complex $\mathscr{S}(B)$ determines a compact polyhedron, as follows. Write $B=\left\{b_{1}, \ldots, b_{t}\right\}$, for an integer $t \geqslant 0$, and let $E=\left\{e_{1}, \ldots, e_{t}\right\}$ be the standard basis of the real vector space $\mathbb{R}^{t}$. Consider the (Euclidean) simplicial complex $\mathscr{E}(B)$ with set of vertices $E$ such that $\left\{e_{i_{1}}, \ldots, e_{i_{u}}\right\}$ spans a simplex of $\mathscr{E}(B)$ if and only if $\left\{b_{i_{1}}, \ldots, b_{i_{u}}\right\} \in \mathscr{S}(B)$, i.e. if and only if $\bigwedge_{j=1}^{u} b_{i_{j}} \neq 0$ in $G$. Then $\bigcup \mathscr{E}(B) \subseteq \mathbb{R}^{t}$ is the underlying polyhedron of $\mathscr{S}(B)$, written $|\mathscr{S}(B)|$. Finally, recall that two finite, abstract simplicial complexes $\mathscr{S}$ and $\mathscr{T}$ with sets of vertices $V$ and $W$, respectively, are isomorphic if there is a bijection $f: V \rightarrow W$ such that $K \in \mathscr{S}$ if and only if $f(K)=\{f(k) \mid k \in K\} \in \mathscr{T}$ for all simplices $K$ of $\mathscr{S}$. In this case, it follows that $|\mathscr{S}|$ and $|\mathscr{T}|$ are piecewise-linearly homeomorphic polyhedra [28, Lemma 2.18].

Corollary. (i) Let $G, G^{\prime}$ be lattice-ordered Abelian groups with finite, regular sets of positive generators $B$ and $B^{\prime}$, respectively. Then $G$ and $G^{\prime}$ are $\ell$-isomorphic if and only if the abstract simplicial complexes $\mathscr{S}(B)$ and $\mathscr{S}\left(B^{\prime}\right)$ have piecewise-linearly homeomorphic underlying polyhedra.

(ii) For any finite abstract simplicial complex $\mathscr{S}$ there exists a lattice-ordered Abelian group $G_{\mathscr{S}}$ with a finite, regular set of positive generators $B \subseteq G_{\mathscr{S}}$ such that $\mathscr{S}(B)$ is isomorphic to $\mathscr{S}$.

This corollary is also proved in Section 2 ,

Remark. The equivalence in (i) of the Corollary fails if the regularity assumption is dropped. For instance, in the ordered subgroups $G$ and $G^{\prime}$ of $\mathbb{R}$ generated by $B=\{1, \sqrt{2}\}$ and $B^{\prime}=\{1,2\}$, respectively, $\mathscr{S}(B)$ and $\mathscr{S}\left(B^{\prime}\right)$ both triangulate a segment, but $G$ and $G^{\prime}$ are not $\ell$-isomorphic - the former has rank 2, while the latter (which is, of course, just $\mathbb{Z}$ ) has rank 1.

In Section 3 we offer three applications of our main results.

In Subsection 3.1 we obtain a novel characterisation of free finitely generated $\ell$-groups in terms of the existence of generating sets with prescribed properties. It may be instructive to compare it to [22] and [25], two previous characterisations. The main difference is that our Corollary 3.1 below is more elementary, in that it mentions neither lattice-group homomorphisms to $\mathbb{Z}$ nor maximal congruences, whereas 222] uses the former and 25] the latter. 
There is a classification to within $\ell$-isomorphism of projective $\ell$-groups with two generators due to Bleier [8, Thm. 3.3] and Beynon [6, p. 252]. It follows at once from the Corollary and the Theorem above, but also from [6, Cor. 3], that the general classification problem for finitely generated projective $\ell$-groups is equivalent to the classification of all compact polyhedra up to piecewise linear homeomorphism. This fact was used in 15 to establish the recursive insolubility of the isomorphism problem for finitely presented $\ell$-groups. By the same token, however, it is in principle possible to obtain complete isomorphism invariants in the language of $\ell$-groups for classes of finitely generated projective $\ell$-groups that correspond to low-dimensional polyhedra. We carry out this programme in Subsection 3.2 for the class of finitely generated projective $\ell$-groups whose maximal spectral space is a closed topological surface. Because of the Corollary, the two needed topological invariants - namely, the Euler characteristic and the orientability class of the maximal spectral space can be algebraically defined in terms of any finite, regular set of positive generators. Besides the classification of surfaces, the proof uses Radó's Triangulation Theorem for 2-dimensional manifolds.

It was proved in [16] that the underlying group of any finitely generated Abelian $\ell$-group is free Abelian of countable rank. The proof uses the classical result of Baer that any countable subgroup of the direct product of a countable number of copies of $\mathbb{Z}$ must be free [2]. The argument in [16] cannot be used to construct a specific $\mathbb{Z}$-module basis for a given finitely generated $\ell$-group. In [26], a $\mathbb{Z}$-module basis of the free 2 -generator Abelian $\ell$-group was exhibited for the first time. In Subsection 3.3 we give a uniform procedure to explicitly construct $\mathbb{Z}$-module bases for any finitely generated projective $\ell$-group, starting from any finite, regular set of positive generators. The proof makes no use of Baer's theorem.

\section{Proof of Theorem and Corollary}

2.1. Preliminaries. In this subsection we recall some notions needed in the sequel, with the purpose of fixing notation and terminology.

Any $\ell$-group is torsion free as a group, and distributive as a lattice [7, 1.2.13, 1.2.14]. Since $\ell$-groups form a finitary algebraic category, arbitrary limits and colimits of $\ell$-groups exist; we shall have occasion to use products. The product $\prod_{i \in I} G_{i}$ of a family $\left\{G_{i}\right\}_{i \in I}$ of $\ell$-groups, where $I$ is any index set, can be described as the group-theoretic direct product of $\left\{G_{i}\right\}_{i \in I}$, with order defined pointwise: $g \in \prod_{i \in I} G_{i}$ satisfies $g \geqslant 0$ if and only if $p_{i}\left(x_{i}\right) \in G_{i}$ satisfies $p_{i}\left(x_{i}\right) \geqslant 0$ in $G_{i}$, for each $i \in I$, where $p_{i}: \prod_{i \in I} G_{i} \rightarrow G_{i}$ is the $i^{\text {th }}$ projection of the group-theoretic direct product $\prod_{i \in I} G_{i}$. Traditionally, the $\ell$-group $\prod_{i \in I} G_{i}$ is called the cardinal product of the family of $\ell$-groups $\prod_{i \in I} G_{i}$, cf. [11, Def. 16.2]. If $I=\emptyset$, then $\prod_{i \in I} G_{i}$ is the terminal object in the category of $\ell$-groups, namely, the trivial singleton $\ell$ group $\{0\}$. By a cardinal factor of the $\ell$-group $G$ we mean any $\ell$-group $A$ such that $G \cong_{\ell} A \times G^{\prime}$ for some $\ell$-group $G^{\prime}$. Here, one can always take $A$ to be the trivial $\ell$-subgroup (=sublattice subgroup) $\{0\} \subseteq G$; when $A$ is not trivial, it is a proper cardinal factor of $G$. If $G$ has no proper cardinal factors, it is cardinally indecomposable.

Any Abelian $\ell$-group has a subdirect representation as a subdirect product of totally ordered groups [7, 4.1.8]. Recall that an $\ell$-subgroup $C$ of an $\ell$-group $G$ is an $\ell$-ideal if $C$ is order-convex, i.e. if $x \leqslant y \leqslant z$ and $x, z \in C$ imply $y \in C$. Kernels of $\ell$ homomorphisms are then precisely $\ell$-ideals, and the usual homomorphism theorems 
hold. The $\ell$-ideals $\mathfrak{p}$ such that the quotient $G / \mathfrak{p}$ is totally ordered are said to be prime. An $\ell$-ideal of the $\ell$-group $G$ is maximal if it is proper (i.e. $\neq G$ ) and inclusion-maximal. Any maximal $\ell$-ideal is automatically prime. The subdirect representation of $G$ can be obtained by embedding $G$ into the cardinal product $\prod G / \mathfrak{p}$, as $\mathfrak{p}$ ranges over all prime $\ell$-ideals of $G$, via the natural map $g \mapsto(g / \mathfrak{p})$.

In any totally ordered Abelian group $G$, if $x, y$ are non-negative elements with $x \geqslant y$, then $(x-(x \wedge y)) \wedge(y-(x \wedge y))=(x-y) \wedge 0=0$. Hence, by subdirect representation: Let $G$ be any Abelian $\ell$-group, and let $x, y \in G^{+}$. Then

$$
(x-(x \wedge y)) \wedge(y-(x \wedge y))=0 .
$$

A concrete model of finitely generated free $\ell$-groups is as follows. Fix an integer $n \geqslant$ 0 , and call a function $f: \mathbb{R}^{n} \rightarrow \mathbb{R}$ piecewise homogeneous linear if $f$ is continuous (with respect to the Euclidean topology of $\mathbb{R}^{n}$ and $\mathbb{R}$ ) and there is a finite number of (homogeneous) linear functions $l_{1}, \ldots, l_{u}: \mathbb{R}^{n} \rightarrow \mathbb{R}$ such that for each $x \in \mathbb{R}^{n}$ we have $f(x)=l_{i}(x)$ for some $i \in\{1, \ldots, u\}$. If the coefficients of each $l_{i}$ can be taken to be integers, then $f$ is integral. Let $\mathscr{A}_{n}$ denote the collection of all integral piecewise homogeneous linear functions $f: \mathbb{R}^{n} \rightarrow \mathbb{R}$. It can be shown that $\mathscr{A}_{n}$ is an $\ell$-group under pointwise addition and order. It is known that $\mathscr{A}_{n}$ is freely generated by the projection functions $\pi_{i}: \mathbb{R}^{n} \rightarrow \mathbb{R}, \pi_{i}\left(x_{1}, \ldots, x_{n}\right)=x_{i}, i=1, \ldots, n$ [4, 6.

A simplicial cone of dimension $m$ in $\mathbb{R}^{n}$ is the non-negative hull $\sigma=\mathbb{R}^{+} v_{1}+$ $\cdots+\mathbb{R}^{+} v_{m}$ of linearly independent vectors $v_{1}, \ldots, v_{m} \in \mathbb{R}^{n}$. We always assume that simplicial cones are non-empty; the non-negative hull of the empty set is $\{0\}$. The (strictly) positive hull of $v_{1}, \ldots, v_{m} \in \mathbb{R}^{n}$ is called the relative interior of $\sigma$. The simplicial cone $\sigma$ is unimodular (also called regular) if the $v_{1}, \ldots, v_{m}$ can be chosen to lie in $\mathbb{Z}^{n}$ in such a way that there exist $w_{m+1}, \ldots, w_{n} \in \mathbb{Z}^{n}$ so that $\left\{v_{1}, \ldots, v_{m}, w_{m+1}, \ldots, w_{n}\right\}$ is a $\mathbb{Z}$-module basis of $\mathbb{Z}^{n}$. Equivalently, the $n \times n$ integer matrix with columns $v_{1}, \ldots, v_{m}, w_{m+1}, \ldots, w_{n}$ has determinant \pm 1 . In this case, the vectors $\left\{v_{1}, \ldots, v_{m}\right\}$ must be primitive: the coordinates of each $v_{i}$ are relatively prime. The non-negative hull of a subset of size $k \geqslant 0$ of $\left\{v_{1}, \ldots, v_{m}\right\}$ is a $k$-dimensional face of $\sigma$, and is itself a unimodular cone. A unimodular fan (also called regular fan) in $\mathbb{R}^{n}$ is a finite collection of unimodular cones $\Delta=\left\{\tau_{1}, \ldots, \tau_{u}\right\}$ such that $\tau_{i} \cap \tau_{j}$ is a common face of $\tau_{i}$ and $\tau_{j}$ for each $i \neq j$. The support, or underlying polyhedron, of the unimodular fan $\Delta$ is the set $|\Delta|=\bigcup_{i=1}^{u} \tau_{i} \subseteq \mathbb{R}^{n}$. If $|\Delta|=\mathbb{R}^{n}$, then $\Delta$ is complete.

Let $\Delta$ be a unimodular fan in $\mathbb{R}^{n}$, and let $\tau \in \Delta$ be a cone. Then the open star of $\tau$ in $\Delta$ is the set of cones of $\Delta$ that contain $\tau$; the star of $\tau$ in $\Delta$ is the closure of the open star of $\tau$ in $\Delta$ under taking faces; and the link of $\tau$ in $\Delta$ is the set-theoretic difference of the star and the open star of $\tau$ in $\Delta$. Now assume $\tau$ is 2 -dimensional, and let $v_{1}, v_{2}$ be the two primitive vectors that span the 1-dimensional faces of $\tau$. Then the (regular) stellar subdivision of $\Delta$ at $\tau$ is the operation of replacing every cone $\sigma$ of $\Delta$ that contains the vector $w=v_{1}+v_{2}$ with the set of all cones of the form

$$
\operatorname{pos}\left(\sigma^{\prime} \cup\{w\}\right),
$$

where $\sigma^{\prime}$ is any face of $\sigma$ that does not contain $w$, and pos denotes the non-negative hull. The result of this operation is a new unimodular fan $\Delta_{v_{1}, v_{2}}$, also called the (regular) stellar subdivision of $\Delta$ at $\left\{v_{1}, v_{2}\right\}$, that satisfies $|\Delta|=\left|\Delta_{v_{1}, v_{2}}\right|$. For 
further background on unimodular fans and regular stellar operations, we refer to [27, 13 .

Let $\Delta$ be a complete unimodular fan in $\mathbb{R}^{n}$, and let $\sigma_{1}, \ldots, \sigma_{m}$ be the list of its 1-dimensional cones. Then each $\sigma_{i}$ is the positive span of a uniquely determined primitive vector $v_{i} \in \mathbb{Z}^{n}$. For each $i=1, \ldots, m$, define a function $h_{i}:|\Delta| \rightarrow \mathbb{R}$ by the conditions (i) $h_{i}\left(v_{i}\right)=1$, (ii) $h_{i}\left(v_{j}\right)=0$ if $i \neq j$, (iii) $h_{i}$ is (homogeneous) linear over each cone of $\Delta$. Using (i)-(iii) and the unimodularity of $\Delta$ one can prove that $h_{i} \in \mathscr{A}_{n}$. The function $h_{i}$ is known as the Schauder hat over $\Delta$ at the 1-dimensional cone $\sigma_{i}$, or at the primitive vector $v_{i}$. The set $H=\left\{h_{1}, \ldots, h_{m}\right\}$ is known as the Schauder basis over $\Delta$.

If $\Delta$ is a unimodular fan in $\mathbb{R}^{n}$, but it is not necessarily complete, a function $f:|\Delta| \rightarrow \mathbb{R}^{n}$ is piecewise homogeneous linear if it is the restriction to $\Delta$ of an element of $\mathscr{A}_{n}$. Let $\mathscr{A}_{n} \uparrow|\Delta|$ be the collection of all elements of $\mathscr{A}_{n}$ restricted to $|\Delta|$; to simplify notation, in the rest of this paper we write $\mathscr{A}_{n} \uparrow \Delta$ in lieu of $\mathscr{A}_{n} \uparrow|\Delta|$. It can be shown that $\mathscr{A}_{n} \uparrow \Delta$ is an $\ell$-group under pointwise operations. In fact, $\mathscr{A}_{n} \uparrow \Delta$ is naturally $\ell$-isomorphic to the quotient $\mathscr{A}_{n} / \mathbb{I}(|\Delta|)$, where $\mathbb{I}(|\Delta|)$ is the $\ell$-ideal of all elements of $\mathscr{A}_{n}$ vanishing over $|\Delta|$. Finitely generated $\ell$-ideals are easily shown to be principal. It can be proved that $\mathbb{I}(|\Delta|)$ is principal, hence $\mathscr{A}_{n} / \mathbb{I}(|\Delta|)$ is projective by [6, Thm. 3.1]; also, each projective quotient of $\mathscr{A}_{n}$ is of this form. Indeed, say $\mathfrak{w}$ is a principal $\ell$-ideal of $\mathscr{A}_{n}$ generated by $w \in \mathscr{A}_{n}$. Then $\mathfrak{w}$ consists of all elements $f \in \mathscr{A}_{n}$ such that there is an integer $m \geqslant 0$ with $m|w| \geqslant|f|$, where $|f|=f \vee-f$. If now $f^{-1}(0)$ denotes the vanishing locus of $f$, i.e. the set $\left\{p \in \mathbb{R}^{n} \mid f(p)=0\right\}$, there is a natural $\ell$-isomorphism $\mathscr{A}_{n} / \mathfrak{w} \cong \mathscr{A}_{n}\left\lceil w^{-1}(0)\right.$, and $w^{-1}(0)$ coincides with the support of some unimodular fan $\Delta$ in $\mathbb{R}^{n}$. For further details, please see [3, 5, 6. Summing up, as $n$ ranges over the non-negative integers, and $\Delta$ over the unimodular fans in $\mathbb{R}^{n}$, to within $\ell$-isomorphism $\mathscr{A}_{n} \uparrow \Delta$ is the most general finitely generated projective $\ell$-group, or equivalenty, the most general quotient of $\mathscr{A}_{n}$ modulo a principal $\ell-i d e a l$. Now if the Schauder basis $H$ over $\Delta$ is defined by the obvious adaptation of the complete case, we have $H \subseteq \mathscr{A}_{n} \uparrow \Delta$.

Let $G$ be an $\ell$-group, and let $B=\left\{b_{1}, \ldots, b_{t}\right\} \subseteq G$ be a finite subset. Following [20. Def. 2.1], we say that $B$ is an abstract Schauder basis of $G$ if there exist an integer $n \geqslant 0$, a unimodular fan $\Delta$ in $\mathbb{R}^{n}$, and an $\ell$-isomorphism $\phi: G \cong_{\ell} \mathscr{A}_{n} \uparrow \Delta$, such that $\phi(B)$ is the Schauder basis over $\Delta$. In short, abstract Schauder bases are what remains of Schauder bases to within $\ell$-isomorphism. The ensuing theory is developed in 20 .

The collection of maximal $\ell$-ideals of $G$, denoted MaxSpec $G$, can be topologised via the the hull-kernel topology whose closed sets are precisely those of the form

$$
\bigcap_{g \in S}\{\mathfrak{m} \in \operatorname{MaxSpec} G \mid g \in \mathfrak{m}\}
$$

as $S$ ranges over the subsets of $G$. For any $\ell$-group $G$, this makes MaxSpec $G$ into a Hausdorff space, known as the maximal spectrum (or the maximal spectral space) of $G$. If, moreover, $G$ has a strong order unit - a non-negative element whose nonnegative multiples are eventually larger than any given $g \in G^{+}-$then MaxSpec $G$ is compact. In particular, whenever $G$ is finitely generated (by $g_{1}, \ldots, g_{u}$, say) it admits a strong order unit (namely, $\sum_{i=1}^{u}\left|g_{i}\right|$ ), and thus its maximal spectrum is a compact Hausdorff space. Note that if $G$ is finitely generated, then MaxSpec $G=\emptyset$ if and only if $G \cong_{\ell}\{0\}$. Further information about spaces of maximal and prime $\ell$-ideals can be found in [7]. 


\subsection{Proof of Theorem.}

Lemma 2.1. Any abstract Schauder basis of an Abelian $\ell$-group $G$ is a finite, regular set of positive generators of $G$.

Proof. Let $B=\left\{b_{1}, \ldots, b_{t}\right\}$ be the basis in question. By definition, there is an integer $n \geqslant 0$, a unimodular fan $\Delta$ in $\mathbb{R}^{n}$, and an $\ell$-isomorphism $\phi: G \rightarrow \mathscr{A}_{n} \uparrow \Delta$, such that $\phi(B)=H$, where $H=\left\{h_{1}, \ldots, h_{t}\right\}$ is the Schauder basis over $\Delta$. It suffices to prove that the finite set $H$ of positive elements is regular and generates $G$, because the latter properties are evidently preserved by $\ell$-isomorphisms.

That $H$ generates $\mathscr{A}_{n} \uparrow \Delta$ is proved in [20, Prop. 2.5]. We only need show that $H$ is regular. For each $h_{i}$, let $\sigma_{i}$ be the 1-dimensional cone of $\Delta$ such that $h_{i}$ is the Schauder hat at $\sigma_{i}$. Further, let $v_{i}$ be the unique primitive vector contained in $\sigma_{i}$, so that $\operatorname{pos} v_{i}=\sigma_{i}$. Given $h_{r} \neq h_{s} \in H$, observe that $H$ is starrable at $\left\{h_{r}, h_{s}\right\}$ if and only if $h_{r} \wedge h_{s}>0$ if and only if $\sigma_{r}$ and $\sigma_{s}$ span a 2-dimensional cone of $\Delta$, say $\tau$. The stellar subdivision of $H$ at $\left\{h_{r}, h_{s}\right\}$ produces a list of elements

$$
\begin{aligned}
h_{r}^{\prime} & =h_{r}-\left(h_{r} \wedge h_{s}\right), \\
h_{s}^{\prime} & =h_{s}-\left(h_{r} \wedge h_{s}\right), \\
h_{i}^{\prime} & =h_{i} \text { for } 1 \leqslant i \leqslant t, r \neq i \neq s, \\
h_{t+1}^{\prime} & =h_{r} \wedge h_{s} .
\end{aligned}
$$

By direct inspection, (国) displays precisely the elements of the Schauder basis $H^{\prime}=$ $\left\{h_{i}^{\prime}\right\}_{i=1}^{t+1}$ over the unimodular fan $\Delta^{\prime}$ obtained by a (regular) stellar subdivision of $\Delta$ at $\tau$ (cf. 20, Lemma 2.4]). The new 1-dimensional cone associated to $h_{t+1}^{\prime}=$ $h_{r} \wedge h_{s}$ is, by definition, the cone $\sigma_{t+1}$ positively spanned by the primitive vector $v_{t+1}=v_{r}+v_{s}$. Let us verify that $H$ is 1 -regular. Fix indices $1 \leqslant i_{1}<\cdots<i_{k} \leqslant t$, and suppose that

$$
\left(h_{r} \wedge h_{s}\right) \wedge h_{i_{1}} \wedge \cdots \wedge h_{i_{k}}>0 \text { holds in } \mathscr{A}_{n} \uparrow \Delta .
$$

Since $H$ is the Schauder basis over $\Delta$, (2) is equivalent to the condition that

$$
\left\{v_{r}, v_{s}, v_{i_{1}}, \ldots, v_{i_{k}}\right\} \text { spans a cone in } \Delta .
$$

By the very definition of (regular) stellar subdivision of $\Delta$ at $\tau$, then, (3) entails that, for every $J \subseteq\left\{i_{1}, \ldots, i_{k}\right\}$ with $\{r, s\} \nsubseteq J$,

$$
\left\{v_{t+1}=v_{r}+v_{s}\right\} \cup\left\{v_{j} \mid j \in J\right\} \text { spans a cone in } \Delta^{\prime} .
$$

In turn, from the definition of Schauder basis over $\Delta^{\prime}$, (44) is equivalent to the condition that, for every $J \subseteq\left\{i_{1}, \ldots, i_{k}\right\}$ with $\{r, s\} \nsubseteq \subseteq J$,

$$
\left(h_{t+1}^{\prime}\right) \wedge \bigwedge_{j \in J} h_{j}^{\prime}>0 \text { holds in } \mathscr{A}_{n} \uparrow \Delta .
$$

Now (5) together with (四) proves that $H$ is 1-regular.

It remains to show that $H^{\prime}$ is 1-regular, too; but this is proved by exactly the same argument as the one just given for $H$, recalling that $H^{\prime}$ is the Schauder basis over $\Delta^{\prime}$.

Lemma 2.2. Let $G$ be an $\ell$-group, and let $B=\left\{b_{1}, \ldots, b_{t}\right\} \subseteq G^{+} \backslash\{0\}$ be a set of positive generators of $G$, for some integer $t \geqslant 0$. If $B$ is 1 -regular, and it is starrable at $\left\{b_{r}, b_{s}\right\}, 1 \leqslant r<s \leqslant t$, then the stellar subdivision $B_{b_{r}, b_{s}}$ has cardinality $t+1$. 
Proof. We have $b_{r} \neq b_{s}$ and $b_{r} \wedge b_{s}>0$ by the starrability assumption. As per ( $*$ ), the stellar subdivision of $B$ at $\left\{b_{s}, b_{r}\right\}$ produces the elements

$$
\begin{aligned}
b_{r}^{\prime} & =b_{r}-\left(b_{r} \wedge b_{s}\right), \\
b_{s}^{\prime} & =b_{s}-\left(b_{r} \wedge b_{s}\right), \\
b_{i}^{\prime} & =b_{i} \text { for } 1 \leqslant i \leqslant t, r \neq i \neq s, \\
b_{t+1}^{\prime} & =b_{r} \wedge b_{s}
\end{aligned}
$$

of $G$. Each element in (i)-(iv) is non-negative in $G$, by construction; we claim that it must in fact be positive. Concerning (iv), we already noted that $b_{t+1}^{\prime} \neq 0$, hence $b_{t+1}^{\prime}>0$ since $b_{r}, b_{s}>0$. For (iii) there is nothing to prove. Finally, for (i)-(ii): if we had, say, $b_{r}-\left(b_{r} \wedge b_{s}\right)=0$, so that $b_{r}=\left(b_{r} \wedge b_{s}\right)$, then $B$ would not be 1-regular, because $\left(b_{r} \wedge b_{s}\right) \wedge b_{r}^{\prime}=0$ whereas $\left(b_{r} \wedge b_{s}\right) \wedge b_{r}=b_{r} \wedge b_{s}>0$, contradicting (R1). This settles the claim.

More generally, the 1-regularity of $B$ implies that there can be no repetitions in the list (i)-(iv). First, it is clear that the elements in (iii) are all distinct, and so are the two elements in (i)-(ii). Here are the remaining three cases.

Case 1 ((i)-(ii) and (iv)). $b_{r}^{\prime}=b_{t+1}^{\prime}$, or $b_{s}^{\prime}=b_{t+1}^{\prime}$.

Suppose $b_{r}^{\prime}=b_{t+1}^{\prime}$, that is, $b_{r}^{\prime}=b_{r} \wedge b_{s}$. Since $b_{r}^{\prime} \wedge b_{s}^{\prime}=0$ by (11), we have $\left(b_{r} \wedge b_{s}\right) \wedge b_{s}^{\prime}=0$. But $\left(b_{r} \wedge b_{s}\right) \wedge b_{s}=b_{r} \wedge b_{s}>0$. This contradicts (R1).

Case 2 ((iii) and (iv)). $b_{i}^{\prime}=b_{t+1}^{\prime}$, for some $1 \leqslant i \leqslant t, r \neq i \neq s$.

Then $B$ is starrable at $\left\{b_{i}, b_{r}\right\}$, because $b_{i} \wedge b_{r}=b_{i}^{\prime} \wedge b_{r}=b_{t+1}^{\prime} \wedge b_{r}=b_{r} \wedge b_{s} \wedge b_{r}=$ $b_{r} \wedge b_{s}>0$. But now the stellar subdivision at $\left\{b_{i}, b_{r}\right\}$ yields $b_{i}-\left(b_{i} \wedge b_{r}\right)=$ $b_{i}-\left(b_{r} \wedge b_{s}\right)=b_{i}^{\prime}-b_{t+1}^{\prime}=0$, whereas we have just shown in the claim above that no stellar subdivision of the regular set $B$ can produce a zero element.

Case 3 ((i)-(ii) and (iii)). $b_{r}^{\prime}=b_{i}^{\prime}$, or $b_{s}^{\prime}=b_{i}^{\prime}$, for some $1 \leqslant i \leqslant t, r \neq i \neq s$.

Suppose $b_{r}^{\prime}=b_{i}^{\prime}$; that is, $b_{r}-\left(b_{r} \wedge b_{s}\right)=b_{i}$. Then $B$ is starrable at $\left\{b_{i}, b_{r}\right\}$, because $b_{i} \wedge b_{r}=\left(b_{r}-\left(b_{r} \wedge b_{s}\right)\right) \wedge b_{r}=b_{r}-\left(b_{r} \wedge b_{s}\right)=b_{r}^{\prime}>0$, by the claim. But now the stellar subdivision at $\left\{b_{i}, b_{r}\right\}$ yields $b_{i}-\left(b_{i} \wedge b_{r}\right)=b_{i}-\left(b_{r}-\left(b_{r} \wedge b_{s}\right)\right)=b_{i}-b_{i}=0$, contradicting the claim above.

We have thus proved that the set

$$
B_{b_{r}, b_{s}}=\left(\left(B \backslash\left\{b_{r}, b_{s}\right\}\right) \cup\left\{b_{r}^{\prime}, b_{s}^{\prime}, b_{t+1}^{\prime}\right\}\right) \backslash\{0\}=\left\{b_{1}^{\prime}, \ldots, b_{t+1}^{\prime}\right\}
$$

has cardinality $t+1$.

Definition 2.3. Let $G$ be an $\ell$-group, and let $B=\left\{b_{1}, \ldots, b_{t}\right\} \subseteq G^{+} \backslash\{0\}$ be a set of positive generators of $G$, for some integer $t \geqslant 0$. Further let $\Sigma$ be a unimodular fan in $\mathbb{R}^{n}$, for some integer $n \geqslant 0$. Consider the $\ell$-group $\mathscr{A}_{n}\lceil\Sigma$, and let $H_{\Sigma}=\left\{h_{1}, \ldots, h_{t^{\prime}}\right\}$ be the Schauder basis over $\Sigma$. An $\ell$-homomorphism $\psi: \mathscr{A}_{n}\lceil\Sigma \rightarrow G$ is said to match $\Sigma$ and $B$ if the following conditions are satisfied:

(M1) $t=t^{\prime}$, and $\psi\left(h_{i}\right)=\psi\left(b_{i}\right)$ for each $i=1, \ldots, t$.

(M2) The map $h_{i} \mapsto b_{i}$, which is a bijection by (M1), is an isomorphism of the abstract simplicial complexes $\mathscr{S}\left(H_{\Sigma}\right)$ and $\mathscr{S}(B)$.

Remark. Observe that (M1) entails that $\psi$ is onto $G$, because $H_{\Sigma}$ generates $\mathscr{A}_{n} \uparrow \Sigma$ [20. Prop. 2.5], and $B$ generates $G$. Moreover, since any $\ell$-homomorphism preserves meets, (M1) implies that the bijection in (M2) carries any simplex of $\mathscr{S}(B)$ to a simplex of $\mathscr{S}\left(H_{\Sigma}\right)$; and thus (M2) amounts to asking that the converse also hold. 
Lemma 2.4. With the same notation as in Definition 2.3, suppose an $\ell$-homomorphism $\psi: \mathscr{A}_{n} \uparrow \Sigma \rightarrow G$ matches $\Sigma$ and $B$. Let $\Sigma^{*}$ be obtained from $\Sigma$ via a finite number $N \geqslant 0$ of (regular) stellar subdivisions. Then, if $B$ is regular, there exists a set $B^{*} \subseteq G^{+} \backslash\{0\}$ obtained from $B$ via $N$ stellar subdivisions in $G$ such that $\psi: \mathscr{A}_{n}\left\lceil\Sigma^{*} \rightarrow G\right.$ matches $\Sigma^{*}$ and $B^{*}$.

Proof. By induction on $N$. If $N=0$, there is nothing to prove. For the induction step, after relabelling it suffices to prove the statement for a single (regular) stellar subdivision applied to $\Sigma$, producing the refined unimodular fan $\Sigma^{*}$. Let $H=H_{\Sigma}=$ $\left\{h_{1}, \ldots, h_{t}\right\}$ be the Schauder basis over $\Sigma$. Arguing exactly as in Lemma 2.1 we see that $H_{\Sigma^{*}}$, the Schauder basis over $\Sigma^{*}$, coincides with $H_{h_{r}, h_{s}}$, the stellar subdivision of $H$ at $\left\{h_{r}, h_{s}\right\}$ in $\mathscr{A}_{n} \uparrow \Sigma$, for a unique choice of indices $r, s \in\{1, \ldots, t\}$. The elements of $H_{h_{r}, h_{s}}$ are displayed as in (E) of Lemma 2.1

Now observe that $b_{r} \wedge b_{s}>0$, because $\psi$ matches $\Sigma$ and $B$, and thus $B$ is starrable at $\left\{b_{r}, b_{s}\right\}$. Set $B^{*}=B_{b_{r}, b_{s}}$, the stellar subdivision of $B$ at $\left\{b_{r}, b_{s}\right\}$, as defined by (*). Then $B^{*}$ has cardinality $t+1$ by Lemma 2.2. Further, comparing (*) with (国), we see that $\psi\left(h_{i}^{\prime}\right)=b_{i}^{\prime}$ for each $i=1, \ldots, t+1$. This shows that (M1) holds.

To prove (M2) it is sufficient to show the following (cf. the Remark after Definition 2.3): for any $1 \leqslant i_{1}<\cdots<i_{k} \leqslant t+1$, if

$$
h_{i_{1}}^{\prime} \wedge \cdots \wedge h_{i_{k}}^{\prime}>0
$$

then

$$
b_{i_{1}}^{\prime} \wedge \cdots \wedge b_{i_{k}}^{\prime}>0
$$

To this aim, set

$$
I=\{r, s, t+1\} \cap\left\{i_{1}, \ldots, i_{k}\right\} .
$$

Note that $\{r, s\} \nsubseteq I$, because $h_{r}^{\prime} \wedge h_{s}^{\prime}=0$ by (1). We argue separately for each of the four remaining cases, using (*) and (因) tacitly.

Case $1 . I=\emptyset$.

Then (6) reads $h_{i_{1}} \wedge \cdots \wedge h_{i_{k}}>0$. By the induction hypothesis we have $b_{i_{1}} \wedge$ $\cdots \wedge b_{i_{k}}>0$, and therefore (7).

Case 2. $I=\{t+1\}$.

Then (6) can be rewritten $h_{i_{1}} \wedge \cdots \wedge h_{i_{k-1}} \wedge\left(h_{r} \wedge h_{s}\right)>0$. By the induction hypothesis we have $b_{i_{1}} \wedge \cdots \wedge b_{i_{k-1}} \wedge\left(b_{r} \wedge b_{s}\right)>0$, and therefore (77).

Case 3. $I=\{r, t+1\}$ or $I=\{s, t+1\}$.

Say $I=\{r, t+1\}$. Then (6) can be rewritten

$$
\left(h_{r} \wedge h_{s}\right) \wedge h_{i_{1}} \wedge \cdots \wedge h_{r}^{\prime} \wedge \cdots \wedge h_{i_{k-1}}>0 .
$$

Since $h_{r} \geqslant h_{r}-\left(h_{r} \wedge h_{s}\right)=h_{r}^{\prime}$, (8) implies

$$
\left(h_{r} \wedge h_{s}\right) \wedge h_{i_{1}} \wedge \cdots \wedge h_{r} \wedge \cdots \wedge h_{i_{k-1}}>0,
$$

which, by the induction hypothesis, yields

$$
\left(b_{r} \wedge b_{s}\right) \wedge b_{i_{1}} \wedge \cdots \wedge b_{r} \wedge \cdots \wedge b_{i_{k-1}}>0 .
$$

Since $B$ is regular, using (R1) with $J=\left\{i_{1}, \ldots, r, \ldots, i_{k}\right\}$, we see that (9) yields

$$
\left(b_{r} \wedge b_{s}\right) \wedge b_{i_{1}}^{\prime} \wedge \cdots \wedge b_{r}^{\prime} \wedge \cdots \wedge b_{i_{k-1}}^{\prime}>0
$$

which is (7). 
Case 4. $I=\{r\}$ or $I=\{s\}$.

Say $I=\{r\}$. Then (6) can be rewritten

$$
h_{i_{1}} \wedge \cdots \wedge h_{r}^{\prime} \wedge \cdots \wedge h_{i_{k}}>0 .
$$

Since $h_{r} \geqslant h_{r}-\left(h_{r} \wedge h_{s}\right)=h_{r}^{\prime}$, (10) implies

$$
h_{i_{1}} \wedge \cdots \wedge h_{r} \wedge \cdots \wedge h_{i_{k}}>0,
$$

which, by the induction hypothesis, yields

$$
b=b_{i_{1}} \wedge \cdots \wedge b_{r} \wedge \cdots \wedge b_{i_{k}}>0 .
$$

We now distinguish two subcases. First, if $\left(b_{r} \wedge b_{s}\right) \wedge b>0$, applying (R1) with $J=\left\{i_{1}, \ldots, r, \ldots, i_{k}\right\}$, we obtain $\left(b_{r} \wedge b_{s}\right) \wedge b_{i_{1}}^{\prime} \wedge \cdots \wedge b_{r}^{\prime} \wedge \cdots \wedge b_{i_{k}}^{\prime}>0$, and $a$ fortiori (7). Otherwise we have $\left(b_{r} \wedge b_{s}\right) \wedge b=0$; equivalently,

$$
b_{s} \wedge b=0 \text {. }
$$

Any Abelian $\ell$-group $G$ has enough primes to yield a subdirect embedding of $G$ into the product $\prod G / \mathfrak{p}$, as $\mathfrak{p}$ ranges over all prime $\ell$-ideals of $G$ [7, 4.1.8]. Therefore, since $b>0$, there exists a prime $\ell$-ideal $\mathfrak{p}$ of $G$ such that $b / \mathfrak{p}>0$ in the totally ordered quotient $G / \mathfrak{p}$. By (12) we must then have

$$
b_{s} / \mathfrak{p}=0 \text {. }
$$

Evaluating $b_{r}^{\prime}$ at $\mathfrak{p}$ using (13), we obtain

$$
b_{r}^{\prime} / \mathfrak{p}=b_{r} / \mathfrak{p}-\left(b_{r} / \mathfrak{p} \wedge b_{s} / \mathfrak{p}\right)=b_{r} / \mathfrak{p} .
$$

From (14), (11), and $b / \mathfrak{p}>0$, we deduce

$$
b / \mathfrak{p}=b_{i_{1}}^{\prime} / \mathfrak{p} \wedge \cdots \wedge b_{r}^{\prime} / \mathfrak{p} \wedge \cdots \wedge b_{i_{k}}^{\prime} / \mathfrak{p}>0 .
$$

But then the element $b^{\prime}=b_{i_{1}}^{\prime} \wedge \cdots \wedge b_{r}^{\prime} \wedge \cdots \wedge b_{i_{k}}^{\prime}$ is non-zero at a prime of $G$, hence it is non-zero in $G$ - and therefore positive in $G$, since we already have $b^{\prime} \geqslant 0$ by assumption. This proves (7).

Construction. Let $G$ be an $\ell$-group, and let $B=\left\{b_{1}, \ldots, b_{t}\right\} \subseteq G^{+} \backslash\{0\}$ be a set of positive generators of $G$, for some integer $t \geqslant 0$. We shall construct a unimodular fan $\Delta_{B}$ in $\mathbb{R}^{t}$ associated to $B$. We write $E=\left\{e_{1}, \ldots, e_{t}\right\}$ for the standard basis of $\mathbb{R}^{t}$. Consider the abstract simplicial complex $\mathscr{S}(B)$ with set of vertices $B$, along with the (Euclidean) simplicial complex $\mathscr{E}(B)$ with set of vertices $E$ such that $\left\{e_{i_{1}}, \ldots, e_{i_{u}}\right\}$ spans a simplex of $\mathscr{E}(B)$ if and only if $\left\{b_{i_{1}}, \ldots, b_{i_{u}}\right\} \in \mathscr{S}(B)$. (Thus $\mathscr{S}(B)$ is defined as in the Introduction, after the statement of the Theorem.) Let $\mathscr{C}$ be the cone over the base $|\mathscr{S}|$ with vertex at the origin of $\mathbb{R}^{t}[28$, p. 2]. Thus, $\mathscr{C}$ is the union of the rays $\mathbb{R}^{+} p$, for each $p \in|\mathscr{S}|$. Then the complex $\mathscr{S}$ uniquely determines a decomposition of $\mathscr{C}$ into a fan $\Delta_{B}$ : the cones of the latter are precisely the cones over the base $T$ with vertex at the origin, as $T$ ranges in $\mathscr{E}(B)$. (The vertex $\{0\}$ of $\Delta_{B}$ is obtained for $T=\emptyset$.) Evidently, $\Delta_{B}$ is unimodular. Observe that the Schauder basis over $\Delta_{B}$ is the set $H_{\Delta_{B}}=\left\{\pi_{1} \uparrow \Delta_{B}, \ldots, \pi_{t} \uparrow \Delta_{B}\right\}$, where $\pi_{i}: \mathbb{R}^{t} \rightarrow \mathbb{R}$ is the $i^{\text {th }}$ projection function, and $\pi_{i} \uparrow \Delta_{B}$ is its restriction to $\left|\Delta_{B}\right|$. We call $\Delta_{B}$ the homogeneous nerve of $B$; cf. P. S. Alexandrov's classical notion of nerve of a system of sets [1, p. 128]. 
Lemma 2.5. Let $G$ be an $\ell$-group, let $B=\left\{b_{1}, \ldots, b_{t}\right\} \subseteq G^{+} \backslash\{0\}$ be a set of positive generators of $G$, for some integer $t \geqslant 0$, and let $\Delta_{B}$ be the homogeneous nerve of $B$ in $\mathbb{R}^{t}$. There is a unique onto $\ell$-homomorphism $\phi_{B}: \mathscr{A}_{t} \uparrow \Delta_{B} \rightarrow G$ that extends the bijection between $H_{\Delta_{B}}$ and $B$ given by $\pi_{i}\left\lceil\Delta_{B} \mapsto b_{i}, i=1, \ldots, t\right.$. Further, this $\ell$-homomorphism $\phi_{B}$ matches $\Delta_{B}$ and $B$.

Proof. Let us define the element $w_{B} \in \mathscr{A}_{t}$ as follows. First, set

$$
O=\bigvee_{i=1}^{t}-\left(\pi_{i} \wedge 0\right) \in \mathscr{A}_{t}
$$

Then, associate with any $\emptyset \neq S \subseteq B$ the element

$$
g_{S}=\bigwedge_{b_{i} \in S} \pi_{i}
$$

of $\mathscr{A}_{t}$. Further, write $\mathscr{Z}$ for the collection of subsets $\emptyset \neq S \subseteq B$ such that $\bigwedge S=0$ in $G$. Finally, set

$$
w_{B}=O \vee\left(\bigvee_{S \in \mathscr{Z}} g_{S}\right) \in \mathscr{A}_{t} .
$$

Now let $\mathfrak{w}_{B}$ be the principal $\ell$-ideal of $\mathscr{A}_{t}$ generated by $w_{B}$, and let $q: \mathscr{A}_{t} \rightarrow$ $\mathscr{A}_{t} / \mathfrak{w}_{B}$ be the canonical quotient map. We claim that there is a unique onto $\ell$-homomorphism $\phi_{B}: \mathscr{A}_{t} / \mathfrak{w}_{B} \rightarrow G$ that extends the function $\pi_{i} / \mathfrak{w}_{B} \mapsto b_{i}, i=$ $1, \ldots, t$. Indeed, consider the following term in the language of $\ell$-groups:

$$
\tau_{B}\left(X_{1}, \ldots, X_{t}\right)=\left(\bigvee_{i=1}^{t}-\left(X_{i} \wedge 0\right)\right) \vee\left(\bigvee_{S \in \mathscr{Z}} \bigwedge_{b_{i} \in S} X_{i}\right),
$$

where $\mathscr{Z}$ is defined as in the preceding paragraph. Let us further write $\tau_{B}\left(b_{1}, \ldots, b_{t}\right)$ to denote the element of $G$ obtained by evaluating the term $\tau_{B}\left(X_{1}, \ldots, X_{t}\right)$ at the tuple of elements $\left(b_{1}, \ldots, b_{t}\right)$ of $G$. Then $\tau_{B}\left(b_{1}, \ldots, b_{t}\right)=0$ holds in $G$ by the very definition of $\tau_{B}\left(X_{1}, \ldots, X_{t}\right)$. Similarly, let $\tau_{B}\left(\pi_{1} / \mathfrak{w}_{B}, \ldots, \pi_{t} / \mathfrak{w}_{B}\right)$ be the element of $\mathscr{A}_{t} / \mathfrak{w}_{B}$ obtained by evaluating $\tau_{B}\left(X_{1}, \ldots, X_{t}\right)$ at the tuple of elements $\left(\pi_{1} / \mathfrak{w}_{B}, \ldots, \pi_{t} / \mathfrak{w}_{B}\right)$ of $\mathscr{A}_{t} / \mathfrak{w}_{B}$. Then, by construction, $\tau_{B}\left(\pi_{1} / \mathfrak{w}_{B}, \ldots, \pi_{t} / \mathfrak{w}_{B}\right)=$ $w_{B}=0$ in $\mathscr{A}_{t}$. In summary, the generating sets $B$ and $\left\{\pi_{1} / \mathfrak{w}_{B}, \ldots, \pi_{t} / \mathfrak{w}_{B}\right\}$ of $G$ and $\mathscr{A}_{t} / \mathfrak{w}_{B}$, respectively, both satisfy the relation $\tau_{B}\left(X_{1}, \ldots, X_{t}\right)=0$. Now since the congruence on $\mathscr{A}_{t}$ determined by the $\ell$-ideal $\mathfrak{w}_{B}$ is generated by the pair $\left(w_{B}, 0\right)$, because $\mathfrak{w}_{B}$ is principal, the $\ell$-group $\mathscr{A}_{t} / \mathfrak{w}_{B}$ is finitely presentable by the single relation $\tau_{B}\left(X_{1}, \ldots, X_{t}\right)=0$, and the latter relation is satisfied by the generators $B$ of $G$, as we just observed. Hence the required extension of $\pi_{i} / \mathfrak{w}_{B} \mapsto b_{i}$ to an onto $\ell$-homomorphism $\phi_{B}: \mathscr{A}_{t} / \mathfrak{w}_{B} \rightarrow G$ exists and is unique by universal algebra; this settles the claim.

Since $\mathfrak{w}_{B}$ is principal, as explained in Subsection 2.1 we can canonically identify the quotient $\mathscr{A}_{t} / \mathfrak{w}_{B}$ with the $\ell$-group $\mathscr{A}_{t} \uparrow V$, where $V=w_{B}^{-1}(0)$ denotes the vanishing locus of the function $w_{B} \in \mathscr{A}_{t}$ in $\mathbb{R}^{t}$. Contemplation of the definiton of $w_{B}$ shows that $V \subseteq \mathbb{R}^{t}$ indeed is the support of the unimodular fan $\Delta_{B}$, the homogeneous nerve of $B$. In fact, the vanishing locus of $O$ in $\mathbb{R}^{t}$ is precisely the non-negative orthant $\mathbb{R}_{\geqslant 0}^{t}$ of $\mathbb{R}^{t}$; that is, for any real numbers $x_{1}, \ldots, x_{t} \in \mathbb{R}, O\left(x_{1}, \ldots, x_{t}\right)=0$ if, and only if, $x_{i} \geqslant 0$, for each $i=1, \ldots, t$. Moreover, $O\left(x_{1}, \ldots, x_{t}\right) \geqslant 0$ for any choice of the $x_{i}$ 's. Therefore, $w_{B}\left(x_{1}, \ldots, x_{t}\right)=0$ implies $x_{i} \geqslant 0$, for each $i=1, \ldots, t$. Further, consider $S=\left\{b_{i_{1}}, \ldots, b_{i_{u}}\right\} \in \mathscr{Z}$. Then $g_{S}^{-1}(0) \cap O^{-1}(0)$ is the complement 
in $\mathbb{R}_{\geqslant 0}^{t}$ of the positive hull of (i.e. the relative interior of the cone spanned by) $\left\{e_{i_{1}}, \ldots, e_{i_{u}}\right\}$, where $\left\{e_{1}, \ldots, e_{t}\right\}$ is the standard basis of $\mathbb{R}^{t}$; hence $V=w_{B}^{-1}(0)$ indeed coincides with $\left|\Delta_{B}\right|$.

Finally, it is obvious by construction that $\phi_{B}$ matches $\Delta_{B}$ and $B$.

Lemma 2.6. Any finite, regular set of positive generators of an Abelian $\ell$-group $G$ is an abstract Schauder basis of $G$.

Proof. Let $B=\left\{b_{1}, \ldots, b_{t}\right\}$ be the set in question, for some integer $t \geqslant 0$. By Lemma 2.5, there is an onto $\ell$-homomorphism

$$
\phi_{B}: \mathscr{A}_{t} \uparrow \Delta_{B} \rightarrow G
$$

that bijects the Schauder basis $H=H_{\Delta_{B}}=\left\{\pi_{1}\left\lceil\Delta_{B}, \ldots, \pi_{t}\left\lceil\Delta_{B}\right\}=\left\{h_{1}, \ldots, h_{t}\right\}\right.\right.$ over the homogeneous nerve $\Delta_{B}$ of $B$ onto the given generating set $B=\left\{b_{1}, \ldots, b_{t}\right\}$, i.e. satisfies $\phi_{B}\left(h_{i}\right)=b_{i}$ for each $i=1, \ldots, t$. Further, $\phi_{B}$ matches $\Delta_{B}$ and $B$. To complete the proof, it suffices to show that (15) is into, and thus is an $\ell$-isomorphism.

Then let $p \in \mathscr{A}_{t} \uparrow \Delta_{B}$ be an element in the kernel of $\phi_{B}$ such that $p \geqslant 0$, with the intent of proving $p=0$. Since $H$ is a Schauder basis, by the abstract De ConciniProcesi Lemma 2 [20, Prop. 5.2], there is a subset $H^{*}=\left\{k_{1}, \ldots, k_{u}\right\} \subseteq \mathscr{A}_{t}\left\lceil\Delta_{B}\right.$ obtained from $H$ via a finite number $N \geqslant 0$ of stellar subdivisions in $\mathscr{A}_{t} \uparrow \Delta_{B}$ such that $p=\sum_{i=1}^{u} p_{i} k_{i}$, for integers $p_{i} \geqslant 0$. Then $H^{*}$ is again a Schauder basis, and it is the Schauder basis over a uniquely determined unimodular fan $\Delta_{B}^{*}$ obtained from $\Delta_{B}$ via $N$ stellar subdivisions. By Lemma 2.4, there is a set $B^{*}=\left\{c_{1}, \ldots, c_{u}\right\} \subseteq$ $G \backslash\{0\}$ that can be obtained from $B$ via $N$ stellar subdivisions, and is such that $\phi_{B}$ matches $\Delta_{B}^{*}$ and $B^{*}$. Thus we have

$$
\phi_{B}\left(k_{i}\right)=c_{i}, i=1, \ldots, u,
$$

and therefore

$$
\phi_{B}\left(k_{i}\right)>0, i=1, \ldots, u \text {. }
$$

Now suppose $p \in \operatorname{ker} \phi_{B}$ satisfies $p>0$, by way of contradiction. Then, in the expansion $p=\sum_{i=1}^{u} p_{i} k_{i}$, there is an index $i_{0}$ such that $p_{i_{0}} \neq 0$. In fact, $p_{i_{0}}>0$ must hold. Let $\sigma_{i_{0}}$ be the unique 1-dimensional cone of $\Delta_{B}^{*}$ such that $k_{i_{0}}$ is the Schauder hat at $\sigma_{i_{0}}$. Further, let $v_{i_{0}}$ be the unique primitive vector contained in $\sigma_{i_{0}}$. By the definition of Schauder basis, $v_{i_{0}}$ has the property that $k_{i_{0}}\left(v_{i_{0}}\right)=1$, whereas $k_{i}\left(v_{i_{0}}\right)=0$ for each $i \neq i_{0}$. If $p_{i_{0}}<0$ holds, then evaluating $p=\sum_{i=1}^{u} p_{i} k_{i}$ at $v_{i_{0}}$ we obtain $p_{i_{0}} k_{i_{0}}\left(v_{i_{0}}\right)=p_{i_{0}}<0$, contradicting our assumption that $p \geqslant 0$.

In summary, there is an index $i_{0}$ such that $p_{i_{0}}>0$. Applying $\phi_{B}$ to $p$, we have $\phi_{B}(p)=\sum_{i=1}^{u} p_{i} \phi_{B}\left(k_{i}\right)$ with $p_{i_{0}}>0$. By (16) we obtain $\phi_{B}(p)>0$, a contradiction. This shows that $\left(\operatorname{ker} \phi_{B}\right)^{+}$is $\{0\}$.

Since any element in ker $\phi_{B}$ can be written as the difference of two non-negative elements in $\operatorname{ker} \phi_{B}$, because $\ell$-groups are directed [7, 1.1.6 and 1.2.11], it follows that $\operatorname{ker} \phi_{B}=\{0\}$, and $\phi_{B}$ is into as was to be shown.

Remark. The Schauder basis over a unmodular fan $\Delta$ in $\mathbb{R}^{n}$ is clearly a linearly independent set (in the $\ell$-group $\mathscr{A}_{n} \uparrow \Delta$, regarded as a $\mathbb{Z}$-module); hence any abstract Schauder basis is, too. It then follows from Lemma 2.6 that regularity indeed

\footnotetext{
${ }^{2}$ To be precise, here we need an equivalent variant of [20, Prop. 5.2] that is easily established: given an abstract Schauder basis $H$ of an $\ell$-group $G$, and given $p \in G^{+}$, there is an abstract Schauder basis $K$ of $G$ such that (a) $p$ lies in the monoidal span of $K$, and (b) $K$ is obtained from $H$ via a finite number of stellar subdivisions.
} 
generalises both pairwise disjointness and linear independence, as was asserted in the Introduction.

Remark. An $\ell$ group $G$ is Archimedean [7, 2.6.1] if, given any $g_{1}, g_{2} \in G$ such that $n g_{1} \leqslant g_{2}$ holds for all integers $n \geqslant 0$, it follows that $g_{1} \leqslant 0$. Note the following non-trivial consequence of Lemma 2.6, any lattice-ordered Abelian group that has a finite, regular set of positive generators must be Archimedean. This is because $\ell$-groups of the form $\mathscr{A}_{t} \uparrow \Delta_{B}$ as in (15) are palpably Archimedean.

2.3. End of Proof of Theorem. By [20, Prop. 4.2], $G$ is finitely generated and projective if, and only if, it has an abstract Schauder basis. By Lemmas 2.1 and 2.6. the latter is the case if, and only if, $G$ has a finite, regular set of positive generators. The Theorem is proved.

2.4. Proof of Corollary. (i) By Lemma 2.6 there are integers $m, n \geqslant 0$, unimodular fans $\Delta$ in $\mathbb{R}^{m}$ and $\Sigma$ in $\mathbb{R}^{n}$, and $\ell$-isomorphisms $\phi: G \rightarrow \mathscr{A}_{m} \uparrow \Delta$ and $\psi: G^{\prime} \rightarrow \mathscr{A}_{n}\left\lceil\Sigma\right.$, such that $\phi(B)=H$ and $\psi\left(B^{\prime}\right)=H^{\prime}$, where $H$ and $H^{\prime}$ are the Schauder bases over $\Delta$ and $\Sigma$, respectively. Following Beynor 3 [6, p. 243], [5. p. 122], we say that a section of (the support of) the fan $\Delta \subseteq \mathbb{R}^{m}$ is a polyhedron $P \subseteq \mathbb{R}^{m}$ such that $P$ does not contain the origin, every ray $\mathbb{R}^{+} v$ from the origin in direction $0 \neq v \in|\Delta|$ meets $P$ in exactly one point, and every ray $\mathbb{R}^{+} v$ from the origin in direction $0 \neq v \notin|\Delta|$ does not meet $P$. It is proved in 6, Cor. 3] that $\mathscr{A}_{m} \uparrow \Delta$ and $\mathscr{A}_{n} \uparrow \Sigma$ are $\ell$-isomorphic if and only if $\Delta$ and $\Sigma$ have piecewise-linearly homeomorphic sections. Let $P$ and $Q$ be sections of $\Delta$ and $\Sigma$, respectively. Since $\phi(B)=H$ and $\psi\left(B^{\prime}\right)=H^{\prime}$, it is clear that there is a piecewise linear homeomorphism from $|\mathscr{S}(B)|$ to $P$, and one from $\left|\mathscr{S}\left(B^{\prime}\right)\right|$ to $Q$. Hence, $G$ and $G^{\prime}$ are $\ell$-isomorphic if and only if $|\mathscr{S}(B)|$ and $\left|\mathscr{S}\left(B^{\prime}\right)\right|$ are piecewise-linearly homeomorphic, as was to be shown.

(ii) Suppose $S=\left\{s_{1}, \ldots, s_{t}\right\}$ is the set of vertices of $\mathscr{S}$. By definition, the polyhedron $|\mathscr{S}|$ comes with a decomposition into a simplicial complex $\mathscr{E}$ on the set of vertices $\left\{e_{1}, \ldots, e_{t}\right\} \subseteq \mathbb{R}^{t}$; namely, $\left\{e_{i_{1}}, \ldots, e_{i_{j}}\right\}$ spans a simplex in $\mathscr{E}$ if and only if $\left\{s_{i_{1}}, \ldots, s_{i_{j}}\right\} \in \mathscr{S}$. Let $\mathscr{C}$ be the cone over the base $|\mathscr{S}|$ with vertex at the origin of $\mathbb{R}^{t}[28$, p. 2]. Then the complex $\mathscr{S}$ uniquely determines a decomposition of $\mathscr{C}$ into a fan $\Delta_{\mathscr{S}}$ : the cones of the latter are precisely the cones over the base $T$ with vertex at the origin, as $T$ ranges in $\mathscr{E}$. Evidently, $\Delta_{\mathscr{S}}$ is unimodular. Let $B$ be the Schauder basis over $\Delta_{\mathscr{S}}$, and set $G_{\mathscr{S}}=\mathscr{A}_{t} \uparrow \Delta_{\mathscr{S}}$. By construction, $B$ is an abstract Schauder basis of $G_{\mathscr{S}}$. By Lemma 2.1 $B$ is a finite, regular set of positive generators of $G_{\mathscr{S}}$.

\section{Applications}

\subsection{A characterisation of finitely generated free $\ell$-groups.}

Corollary 3.1. Fix an integer $n>0$. For any lattice-ordered Abelian group $G$, the following are equivalent.

(1) $G$ is freely generated by $n$ elements.

\footnotetext{
${ }^{3}$ For the sake of precision, let us note that Beynon's original definition of a section is incomplete, as he only asks - with our present notation - that every ray $\mathbb{R}^{+} v$ from the origin in direction $0 \neq v \in|\Delta|$ meets $P$ in exactly one point. On this definition, then, the boundary of the cube $[-1,1]^{m}$ is a section of the support of any non-trivial fan in $\mathbb{R}^{m}$, which is clearly not what is wanted.
} 
(2) $G$ has a regular set $S$ of positive generators such that

(a) $|S|=n+1$,

(b) $\bigwedge S=0$, and

(c) $\bigwedge S^{\prime} \neq 0$ for any proper, non-empty subset $S^{\prime} \subset S$.

Proof. Let us first show that the free $\ell$-group $\mathscr{A}_{n}$ has a regular set $H$ of positive generators satisfying (a)-(c). Let us define a complete fan $\Theta_{n}$ in $\mathbb{R}^{n}$ as follows. The 1-dimensional cones of $\Theta_{n}$ are generated by the standard basis vectors $E=$ $\left\{e_{1}, \ldots, e_{n}\right\}$ along with $e_{0}=-\left(e_{1}+\cdots+e_{n}\right)$; further, any proper subset of $E \cup$ $\left\{e_{0}\right\}$ spans a cone of $\Theta_{n}$ (see Figure 11). A trivial computation shows that $\Theta_{n}$ is unimodular. Let $H$ be the Schauder basis over $\Theta_{n}$. Then $H$ is an abstract Schauder basis of $\mathscr{A}_{n}$. By Lemma 2.1, $H$ is a finite, regular set of positive generators of $\mathscr{A}_{n}$. By construction, $H$ satisfies (a)-(c) in $\mathscr{A}_{n}$. This proves $(1 \Rightarrow 2)$. To prove the converse implication, observe that from (a)-(c) it easily follows that $\mathscr{S}(S)$ is isomorphic to $\mathscr{S}(H)$, where $H$ is the Schauder basis just described, and thus $|\mathscr{S}(S)|$ is piecewise-linearly homeomorphic to $|\mathscr{S}(H)|$. Now apply (i) in the Corollary (in the Introduction).

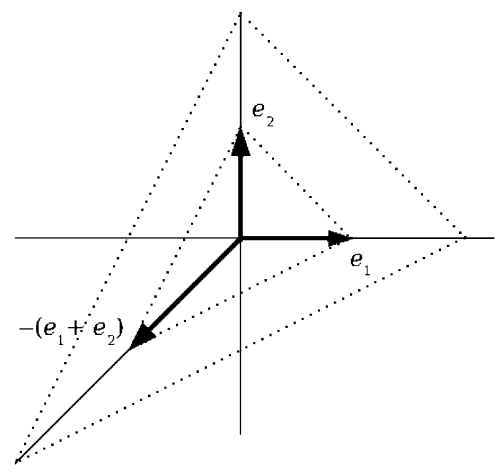

Figure 1. The fan $\Theta_{n}$ in $\mathbb{R}^{n}$ used in the proof of Corollary 3.1, case $n=2$. (The toric variety associated with $\Theta_{2}$ is the complex projective plane; see [27, Example (ii) on p. 9].)

3.2. The Euler characteristic and $\ell$-groups. Recall that a topological space $M$ is locally Euclidean of dimension $n$, for $n \geqslant 1$ an integer, if every point of $M$ has a neighbourhood homeomorphic to an open subset of $\mathbb{R}^{n}$. Such a space $M$ is a manifold (without boundary) of dimension $n$ if it is second-countable and Hausdorff. By a surface we shall mean a compact manifold of dimension 2. For background on the topology of manifolds, see e.g. [18.

Let us say that an $\ell$-group $G$ is an $\ell$-surface if it is finitely generated and projective, and MaxSpec $G$ is a surface. We are going to provide a complete classification of $\ell$-surfaces in elementary algebraic terms, i.e. without explicitly using conditions on maximal spectral spaces. We begin with a reduction to directly indecomposable components of $\ell$-surfaces.

Proposition 3.2. Any finitely generated projective lattice-ordered Abelian group $G$ splits into a finite cardinal product

$$
G \cong G_{1} \times G_{2} \times \cdots \times G_{u},
$$


with each $G_{i}$ a cardinally indecomposable finitely generated projective lattice-ordered Abelian group. Further, such a decomposition is unique (to within $\ell$-isomorphism) up to the order of the factors. Finally, $G$ is an $\ell$-surface if and only if each $G_{i}$ is an $\ell$-surface, for $i=1, \ldots, u$.

Remark. Surely the substance of this proposition must be known to specialists; cf., for instance, the results on product decompositions in [19, Ch. III, §11]. Nonetheless, we know of no complete proof in the literature, and we therefore include one.

Proof. By the Theorem, $G$ has a finite, regular set of generators $B$. If $B=\emptyset$, $G=\{0\}$ and the proposition holds trivially. Assume $B \neq \emptyset$. Say $x, y \in B$ are connected if there is a sequence $x=x_{0}, x_{1}, \ldots, x_{u}=y$ of elements of $B$ such that $x_{i} \wedge x_{i+1}>0$ in $G$. Connectedness is an equivalence relation on $B$ whose associated partition we display as $B_{1}, \ldots, B_{u}$. For each $i=1, \ldots, u$, by the Theorem and Lemma 2.6. $B_{i}$ generates in $G$ a projective $\ell$-group $G_{i}$ of which $B_{i}$ is an abstract Schauder basis. By [20, Prop. 5.2], each $g \in G$ lies in the subgroup of $G$ generated by a stellar subdivision $K$ of $B$. But since each $x \in B_{i}$ is disjoint from each $y \in B_{j}$, for $i \neq j, K$ can be written as a disjoint union $K_{1} \sqcup \cdots \sqcup K_{u}$, where each $K_{i}$ is a stellar subdivision of $B_{i}$. Hence, $g$ lies in the group-theoretic sum $G_{1}+\cdots+G_{u}$. Moreover, by construction, it is clear that each $G_{i}$ is the polar [11, p. 70] of $\bigcup_{j \neq i} B_{j}$. By [11, Cor. 16.4] it follows that each $G_{i}$ is a cardinal factor of $G$, and $G \cong_{\ell} G_{1} \times G_{2} \times \cdots \times G_{u}$. To see that each $G_{i}$ is cardinally indecomposable, suppose $G_{i} \cong \cong_{\ell} F_{1} \times F_{2}$ with $F_{1}$ and $F_{2}$ non-trivial $\ell$-groups. We claim that each $x \in B_{i}$ satisfies $x \in F_{1}$ or $x \in F_{2}$. If that is not the case, since each cardinal factor $F_{1}$ is evidently a polar, $x$ may be uniquely written as $x_{1}+x_{2}, x_{1} \wedge x_{2}=0, x_{1}, x_{2}>0$, and $x_{1} \in F_{1}, x_{2} \in F_{2}$. Represent $B_{i}$ as the Schauder basis over a unimodular fan $\Delta$ in $\mathbb{R}^{m}$ via an $\ell$-isomorphism $\phi: G_{i} \rightarrow \mathscr{A}_{m} \uparrow \Delta$, let $\mathbb{S}_{m-1}$ be the unit sphere in $\mathbb{R}^{m}$, and let $S$ be the support of $\phi(x)$ intersected with $\mathbb{S}_{m-1}$. If we further let $S_{1}$ and $S_{2}$ respectively denote the supports of $\phi\left(x_{1}\right)$ and $\phi\left(x_{2}\right)$ intersected with $\mathbb{S}_{m-1}$, we see that $x_{1}, x_{2}>0$ implies $S_{1} \neq \emptyset \neq S_{2}$, and that $\phi\left(x_{1}\right) \wedge \phi\left(x_{2}\right)=0$ implies $S=S_{1} \sqcup S_{2}$; in other words, $S$ is disconnected as a subspace of $\mathbb{S}_{m-1}$ (the latter with its standard Euclidean topology). But if $h$ is any Schauder hat of the Schauder basis over $\Delta$, it is easily checked that the intersection $O$ of the support of $h$ with $\mathbb{S}_{m-1}$ must be connected, by the very definition of Schauder hat. Indeed, suppose $h$ is the hat at the 1-dimensional cone $\sigma \in \Delta$, and let $O$ and $L$ be the open star and the link of $\sigma$ in $\Delta$, respectively. Then the closure of $O \cap \mathbb{S}_{m-1}$ is $(O \cup L) \cap \mathbb{S}_{m-1}$, and the latter has the same topological type of a cone [28, p. 2] with vertex $p$ and base $L \cap \mathbb{S}_{m-1}$. Since cones are contractible by definition, they certainly are connected. Now $O \cap \mathbb{S}_{m-1}$ on its own is not a cone (because it does not generally have a compact base), but it is itself contractible because it is written as a cone less its compact base. Hence our claim is settled, and $B_{i}$ can be written as a disjoint union of non-empty sets $\left(B_{i} \cap F_{1}\right) \sqcup\left(B_{i} \cap F_{2}\right)$. Since each $x \in\left(B_{i} \cap F_{1}\right)$ is disjoint from each $y \in\left(B_{i} \cap F_{2}\right)$, we conclude that $x$ and $y$ are not connected, a contradiction. Therefore, $G_{i}$ is cardinally indecomposable. Now the uniqueness assertion in the proposition follows from [11, Prop. 16.18].

To prove the last assertion, one checks directly from definitions that MaxSpec $G$ is homeomorphic to the disjoint union MaxSpec $G_{1} \sqcup \cdots \sqcup \operatorname{MaxSpec} G_{n}$, endowed with the coproduct topology having as a base of closed sets the sets of the form $C_{1} \sqcup \cdots \sqcup C_{n}$, where $C_{i}$ ranges in the base of closed sets of MaxSpec $G_{i}$ given by 
the hull-kernel topology. It follows at once that $\operatorname{MaxSpec} G$ is a surface if and only if each MaxSpec $G_{i}$ is, and the proof is complete.

Now let $G$ be a cardinally indecomposable $\ell$-surface $G$, and let $B$ be a finite, regular set of positive generators of $G$. (Such a set always exists by the Theorem.) For each integer $i \geqslant 1$, let $f_{i}$ be the number of distinct subsets $F \subseteq B$ of cardinality $i$ such that $\bigwedge F \neq 0$ in $G$, i.e. such that $F \in \mathscr{S}(B)$. Thus, $f_{1}=|B|$, and the sequence $f_{1}, f_{2}, \ldots$ is eventually zero. We define the Euler characteristic of $G$ to be the integer

$$
\chi_{G}=f_{1}-f_{2}+f_{3}-f_{4}+\cdots .
$$

Further, we say that the cardinally indecomposable $\ell$-surface $G$ is non-orientable if the polyhedron $|\mathscr{S}(B)|$ has trivial integral homology in dimension 2 - that is, if

$$
H_{2}(\mathscr{S}(B), \mathbb{Z}) \cong\{0\} .
$$

Otherwise, we say that $G$ is orientable. For background on the homology of simplicial complexes and the orientability of manifolds, see e.g. [17, Ch. 2 and Thm. 3.26, respectively]. The next proposition is the counterpart for $\ell$-groups of the classical topological invariance of the Euler characteristic and of simplicial homology.

Proposition 3.3. Let $G$ be a cardinally indecomposable $\ell$-surface. Then the Euler characteristic of $G$, as given by $(\mathbb{X})$, and the orientability of $G$, as given by condition $\left(\star^{\prime}\right)$, are well defined, in that they do not depend on the choice of the finite, regular set of generators $B$.

Proof. Let $B^{\prime}$ be another such set of generators for $G$. By (i) in the Corollary we know that $|\mathscr{S}(B)|$ and $\left|\mathscr{S}\left(B^{\prime}\right)\right|$ are homeomorphic, and therefore their simplicial homology groups with coefficients in $\mathbb{Z}$ are isomorphic by e.g. 17, Cor. 2.11 and Thm. 2.27]; in particular, $H_{2}(\mathscr{S}(B), \mathbb{Z})$ is trivial if and only if $H_{2}\left(\mathscr{S}\left(B^{\prime}\right), \mathbb{Z}\right)$ is, and the orientability of $G$ does not depend on the choice of $B$. Similarly, observe that $(\mathbb{X})$ is the formula for the Euler characteristic $\chi$ of the simplicial complex $\mathscr{S}(B)$, and it is a classical fact that $\chi$ only depends on the underlying polyhedron $|\mathscr{S}(B)|$, and not on the chosen triangulation $\mathscr{S}(B)$; for a proof of this fact in the case of surfaces, see e.g [23, Ch. 21, Thm. 5]. Hence, $\chi_{G}$ does not depend on the choice of $B$ by (i) in the Corollary.

Before stating the main classification result of this subsection, we prepare a general lemma.

Lemma 3.4. Let $G$ be a lattice-ordered Abelian group with a finite, regular set of positive generators $B$. Then the maximal spectral space MaxSpec $G$ is homeomorphic to $|\mathscr{S}(B)|$.

Proof. By Lemma 2.6, $B$ is an abstract Schauder basis of $G$. Therefore, there exist an integer $m \geqslant 0$, a unimodular fan $\Delta$ in $\mathbb{R}^{m}$, and an $\ell$-isomorphism $\phi: G \rightarrow$ $\mathscr{A}_{m}\lceil\Delta$, such that $\phi(B)$ is the Schauder basis over $\Delta$. Now it is easy to check that maximal $\ell$-ideals of $\mathscr{A}_{m}$ are in bijection with the rays $\mathbb{R}^{+} v$ in $\mathbb{R}^{m}$, where $0 \neq v \in \mathbb{R}^{m}$. Indeed, given such a ray, the collection of functions in $\mathscr{A}_{m}$ that vanish at $v$ (equivalently, by positive homogeneity, at $\mathbb{R}^{+} v$ ) is a maximal $\ell$-ideal. Conversely, if $\mathfrak{m}$ is a maximal $\ell$-ideal of $\mathscr{A}_{m}$, then its vanishing locus $\mathbb{V}_{\mathfrak{m}}=\{v \in$ $\mathbb{R}^{m} \mid f(v)=0$ for all $\left.f \in \mathfrak{m}\right\}$ must be a ray. To generalise this correspondence to not necessarily maximal $\ell$-ideals of $\mathscr{A}_{m}$, let $\mathbb{S}_{m-1}$ denote the unit sphere in $\mathbb{R}^{m}$, 
with the topology inherited from the Euclidean topology of $\mathbb{R}^{m}$. Let $I$ be an $\ell$ ideal of $\mathscr{A}_{m}$, and consider its vanishing locus $\mathbb{V}_{I}$. It is easy to verify that there is a unique closed subset of $C \subseteq \mathbb{S}_{m-1}$ such that $\mathbb{V}_{I}$ is the cone with base $C$ and vertex at the origin; namely, $C=\mathbb{V}_{I} \cap \mathbb{S}_{m-1}$. Conversely, given a closed subset $C \subseteq \mathbb{S}_{m-1}$, and given $v \in C$, let us write $\mathfrak{m}_{v}$ for the unique maximal $\ell$-ideal of $\mathscr{A}_{m}$ whose vanishing locus is the ray $\mathbb{R}^{+} v$. The intersection $\bigcap_{v \in C} \mathfrak{m}_{v}$ is then an $\ell$-ideal of $\mathscr{A}_{m}$ whose vanishing locus is precisely the cone with base $C$ and vertex at the origin. We conclude that the assignment $\mathfrak{m} \mapsto \mathbb{V}_{\mathfrak{m}} \cap \mathbb{S}_{m-1}$ yields a bijection that is continuous (because it preserves a basis of closed sets), and therefore is a homeomorphism (because its domain MaxSpec $\mathscr{A}_{m}$ is compact, and its codomain $\mathbb{S}_{m-1}$ is Hausdorff). Using the fact that the maximal $\ell$-ideals of $\mathscr{A}_{m} \uparrow \Delta$ are in bijection with the rays $\mathbb{R}^{+} v$ with $v \in|\Delta|$, the argument has a straightforward generalisation that shows that $\operatorname{Max} \operatorname{Spec}\left(\mathscr{A}_{m}\lceil\Delta)\right.$ is homeomorphic to $\mathbb{S}_{m-1} \cap|\Delta|$.

Arguing as in the proof of (ii) in the Corollary, we observe that $|\mathscr{S}(B)|$ is piecewise-linearly homeomorphic to a section $P$ of $\Delta$. But it is seen at once that any such section $P$ is homeomorphic to $\mathbb{S}_{m-1} \cap|\Delta|$, for any point $p \in P$ has a unique radial projection onto the point $\mathbb{R}^{+} p \cap\left(\mathbb{S}_{m-1} \cap|\Delta|\right)$ of $\left(\mathbb{S}_{m-1} \cap|\Delta|\right)$, and conversely. This completes the proof.

We can now prove:

Theorem 3.5 (Classification of cardinally indecomposable $\ell$-surfaces). Let $G$ and $G^{\prime}$ be two cardinally indecomposable $\ell$-surfaces. Then $G$ and $G^{\prime}$ are $\ell$-isomorphic if, and only if, they are either both orientable or both non-orientable, and $\chi_{G}=\chi_{G^{\prime}}$.

Proof. If $\phi: G \rightarrow G^{\prime}$ is an $\ell$-isomorphism, let $B$ be a finite, regular set of positive generators of $G$, which always exists by the Theorem. Then $\phi(B)$ is a finite, regular set of positive generators of $G^{\prime}$, and $\mathscr{S}(B)$ and $\mathscr{S}(\phi(B))$ are isomorphic abstract simplicial complexes. Therefore, $\chi_{G}=\chi_{G^{\prime}}$ and $H_{2}(\mathscr{S}(B), \mathbb{Z})$ is isomorphic to $H_{2}(\mathscr{S}(\phi(B)), \mathbb{Z})$, as was to be shown. For the converse, suppose that $G$ and $G^{\prime}$ are both orientable or both non-orientable, and assume $\chi_{G}=\chi_{G^{\prime}}$. By the Theorem, choose finite, regular sets of generators $B$ and $B^{\prime}$ of $G$ and $G^{\prime}$, respectively. Since $G$ and $G^{\prime}$ are cardinally indecomposable $\ell$-surfaces, by Lemma 3.4 and Proposition 3.2 we infer that $|\mathscr{S}(B)|$ and $\left|\mathscr{S}\left(B^{\prime}\right)\right|$ are connected surfaces. Now the abstract simplicial complexes $\mathscr{S}(B)$ and $\mathscr{S}\left(B^{\prime}\right)$ have the same Euler characteristic and belong to the same orientability class, by our assumptions about $G$ and $G^{\prime}$. By the classification of connected surfaces [23, Thm. 22.9], it follows that $|\mathscr{S}(B)|$ and $\left|\mathscr{S}\left(B^{\prime}\right)\right|$ are homeomorphic. Radó's Triangulation Theorem [23, Thm. 8.3] for 2-dimensional manifolds then implies that $|\mathscr{S}(B)|$ and $\left|\mathscr{S}\left(B^{\prime}\right)\right|$ must be piecewiselinearly homeomorphic [23, Thm. 8.5]. Now from (i) in the Corollary it follows that $G$ and $G^{\prime}$ are $\ell$-isomorphic, and the proof is complete.

Since the 2-dimensional sphere is the only orientable surface having Euler characteristic 2, from Theorem 3.5 we obtain (compare Corollary 3.1):

Corollary 3.6. A lattice-ordered Abelian group $G$ is freely generated by three elements if, and only if, $G$ is a cardinally indecomposable orientable $\ell$-surface with $\chi_{G}=2$.

Proof. It suffices to check that $\mathscr{A}_{3}$ is a cardinally indecomposable orientable $\ell$ surface with $\chi_{G}=2$, and then the result follows from Theorem 3.5. By Corollary 3.1 and Lemma 3.4 we see that MaxSpec $\mathscr{A}_{3}$ is homeomorphic to a 2-dimensional 
sphere. From this it follows easily that $\mathscr{A}_{3}$ is cardinally indecomposable, using the fact that MaxSpec $\mathscr{A}_{3}$ is connected. (Argue along the lines of the last paragraph in the proof of Proposition 3.2.) Hence, $\mathscr{A}_{3}$ is a cardinally indecomposable $\ell$-surface. Computing $\chi_{\mathscr{A}_{3}}$ using the generating set $S$ provided by Corollary 3.1. we see that $\chi_{\mathscr{A}_{3}}=2$. A further computation shows $H_{2}(\mathscr{S}(S), \mathbb{Z}) \cong \mathbb{Z}$.

3.3. $\mathbb{Z}$-module bases of finitely generated projectives. Consider a finite, regular set $B=\left\{b_{1}, \ldots, b_{m}\right\}$ of positive generators of an $\ell$-group $G$. Since $B$, by Lemma 2.6 is an abstract Schauder basis of $G$, it follows that $B$ is linearly independent in the $\mathbb{Z}$-module $G$. Hence the subgroup $[B]$ that $B$ generates in $G$ is free Abelian of rank $n$; choosing $B$ as a $\mathbb{Z}$-module basis of $[B]$ we obtain a group isomorphism $[B] \cong \mathbb{Z}^{m}$.

Let us say that $B$ splits the element $g=z_{1} b_{1}+\cdots+z_{m} b_{m}$ of $[B]$ if whenever $b_{i_{1}}, b_{i_{2}} \in B$ are such that $z_{i_{1}} z_{i_{2}}<0$, then $b_{i_{1}} \wedge b_{i_{2}}=0$ holds in $G$. Unless $B$ is pairwise disjoint, or $G$ is trivial and therefore $B=\emptyset$, there must be elements of $[B]$ that are not split by $B$. However, we can ask that $B$ splits every element of $[B]$ up to subdivisions, in the following sense.

Given a sequence

$$
B=B_{0}, B_{1}, \ldots, B_{n}, \ldots
$$

of subsets of $G$ such that, for $i=1,2, \ldots, B_{i}$ is obtained from $B_{i-1}$ via one stellar subdivision at

$$
\left\{h^{(i-1)}, k^{(i-1)}\right\} \subseteq B_{i-1},
$$

let us say that (†) splits the subgroup $[B]$ if for every element $g \in[B]$ there is an index $j=0,1, \ldots$ such that $B_{j}$ splits $g \in\left[B_{j}\right]$. This makes sense: since $B_{j}$ is obtained from $B$ via a finite number of stellar subdivisions, $B_{j}$ is again a regular set of positive generators of $G$; and it follows from the definition of stellar subdivision that $[B] \subseteq\left[B_{j}\right]$.

Considering again the sequence (†), let us define an associated sequence to be used below, by setting

$$
\begin{aligned}
& \hat{B}_{0}=B \\
& \hat{B}_{1}=\hat{B}_{0} \cup\left\{h^{(0)} \wedge k^{(0)}\right\}
\end{aligned}
$$

$(\hat{\dagger})$

$$
\hat{B}_{i}=\hat{B}_{i-1} \cup\left\{h^{(i-1)} \wedge k^{(i-1)}\right\}
$$

Finally, let us recall that an $\ell$-group $G$ is simplicial if it is $\ell$-isomorphic to the cardinal product of a finite number of copies of $\mathbb{Z}$, the latter with its natural order. In other words, there is a group isomorphism $G \cong \mathbb{Z}^{r}$ for a non-negative integer $r$, and $\left(z_{1}, \ldots, z_{r}\right) \in \mathbb{Z}^{r} \cong G$ is non-negative if and only if $z_{i} \geqslant 0$ for each $i=1, \ldots, r$. (Here, $G$ is the trivial $\ell$-group $\{0\}$ when $r=0$.) By definition, the underlying group of a simplicial group $\mathbb{Z}^{r}$ is free Abelian of finite rank $r$. One can verify that the standard basis of $\mathbb{Z}^{r}$ is the unique regular set of positive generators of $\mathbb{Z}^{r}$ - so that simplicial groups are projective by the Theorem — and that such a set splits every element of $\mathbb{Z}^{r}$. 
After these preparations, we can prove:

Theorem 3.7 (Z्Z-module bases of finitely generated projective Abelian $\ell$-groups). Let $G$ be a lattice-ordered Abelian group. Suppose $G$ has a finite, regular set of positive generators $B$. Then either $G$ is a simplicial group or else the following hold.

(i) There exists a sequence $B=B_{0}, B_{1}, \ldots, B_{n}, \ldots$ of subsets of $G$ such that (a) $B_{i}$ is obtained from $B_{i-1}$ via one stellar subdivision, for $i=1,2, \ldots$, and (b) the sequence splits the subgroup $[B]$ generated by $B$ in $G$.

(ii) Whenever $B=B_{0}, B_{1}, \ldots, B_{n}, \ldots$ is as in (i), and the sequence $\hat{B}_{0}, \hat{B}_{1}$, $\ldots, \hat{B}_{n}, \ldots$ is defined as in $(\hat{\dagger})$, the set

$$
\hat{B}_{\infty}=\bigcup_{i \geqslant 0} \hat{B}_{i}
$$

is a $\mathbb{Z}$-module basis for the $\mathbb{Z}$-module $G$, and consists of positive elements.

In particular, whenever a lattice-ordered Abelian group $G$ is finitely generated and projective, the underlying group of $G$ is free Abelian of countable rank.

Proof. (i) We first observe that, since $G$ is not simplicial, whenever $K$ is obtained from $B$ via a finite number of stellar subdivision, then $K$ must be starrable at some pair $\{h, k\} \in K$. For otherwise $K$ must be a finite, pairwise disjoint set of positive generators of $G$, and this easily entails that $G$ is simplicial, a contradiction. We make use of this observation in constructing the desired sequence $B=B_{0}, B_{1}, \ldots$ by induction. Fix an enumeration $g_{0}=0, g_{1}, g_{2}, \ldots$ of all elements of (the countable set) $[B]$. Then $B$ trivially splits $g_{0}=0$, because $B$ is linearly independent in the $\mathbb{Z}$-module $G$. Assume by induction that a finite sequence $B=B_{0}, B_{1}, \ldots, B_{n}$ has been defined so that each $B_{i}$ is obtained from $B_{i-1}$ via one stellar subdivision, for $i=1, \ldots, n$, and there is an integer $s>0$ such that $B_{n}$ splits each $g_{j}$, $j=0,1, \ldots, s$. To close the induction, it suffices to show that there is a finite extension of $B_{0}, B_{1}, \ldots, B_{n}$ to $B_{0}, B_{1}, \ldots, B_{n}, B_{n+1}, \ldots, B_{n+u}$, with $u \geqslant 1$ an integer, obtained by performing one stellar subdivision at each step and such that $B_{n+u}$ splits $g_{s+1}$. We distinguish two cases.

In the first case, the (non-zero) element $g_{s+1}$ is either positive or negative in $G$. Then all coefficients in the linear expansion of $g$ in terms of $B_{n}$ are $\geqslant 0$ or $\leqslant 0$, respectively. To see this, let us identify $B_{n}$ with the Schauder basis over some unimodular fan $\Delta$ in $\mathbb{R}^{m^{\prime}}$, by virtue of Lemma 2.6. If in the linear expansion of $g$ there occur coefficients $z_{1}$ and $z_{2}$ (for hats $h_{1}$ and $h_{2}$, say, at the primitive vectors $v_{1}$ and $v_{2}$, respectively) with $z_{1} z_{2}<0$, then $g$ is not comparable to 0 : evaluation at $v_{1}$ yields $z_{1}$, and evaluation at $v_{2}$ yields $z_{2}$. Hence, $B_{n}$ splits $g_{s+1}$ in this case. To complete the induction, we can take $u=1$, and let $B_{n+1}$ be any set obtained from $B_{n}$ by one stellar subdivision; such a set exists by our initial observation.

In the second case, $g_{s+1}$ is incomparable to 0 in $G$. If $g_{s+1}$ lies in $\left[B_{n}\right]$ and $B_{n}$ happens to split $g_{s+1}$, we take $u=1$ and let $B_{n+1}$ be any set obtained from $B_{n}$ by one stellar subdivision; again, such a set certainly exists. It is then easy to check that $B_{n+1}$ must split $g_{s+1}$, too, and the induction is complete. On the other hand, suppose that either $g_{s+1}$ does not lie in $\left[B_{n}\right]$, or $B_{n}$ does not split $g_{s+1}$. Then there are uniquely determined positive elements $p=g_{s+1} \vee 0$ and $q=\left(-g_{s+1}\right) \vee 0$ such 
that $g_{s+1}=p-q$, and $p \wedge q=0$. By [20, Prop. 5.2], there exists a subset $B_{n+u}$ obtained from $B_{n}$ via a finite number $u$ of stellar subdivisions such that both $p$ and $q$ lie in the monoidal span of $B_{n+u}$. Then $g_{s+1}$ lies in $\left[B_{n+u}\right]$. Further, $B_{n+u}$ splits $g_{s+1}$. To see this, suppose $B_{n+u}=\left\{b_{1}, \ldots, b_{u}\right\}$, and write $p=\sum_{i=1}^{u} p_{i} b_{i}$, $q=\sum_{i=1}^{u} q_{i} b_{i}$ for uniquely determined integers $p_{i}, q_{i} \geqslant 0$. Since $p \wedge q=0$, for each $i=1, \ldots, u$ we must have $p_{i} q_{i}=0$, or else $b_{i}$ would be a positive lower bound for $\{p, q\}$. Hence, up to a permutation of the indices, there is an index $0<j<u$ such that $p=\sum_{i=1}^{j} p_{i} b_{i}$ and $q=\sum_{i=j+1}^{u} q_{i} b_{i}$. Again because of $p \wedge q=0$, we must have $b_{r} \wedge b_{r^{\prime}}=0$ whenever $r \in\{1, \ldots, j\}$ and $r^{\prime} \in\{j+1, \ldots, u\}$. This shows that $B_{n+u}$ splits $g_{s+1}=p-q$ (so that necessarily $u \geqslant 1$ by our assumption about $g_{s+1}$ ), and completes the proof of (i).

(ii) Since, by Lemma 2.6, $B=\left\{b_{1}, \ldots, b_{m}\right\}$ is an abstract Schauder basis of $G$, there exist an integer $m^{\prime} \geqslant 0$, a unimodular fan $\Delta$ in $\mathbb{R}^{m^{\prime}}$, and an $\ell$-isomorphism $\phi: G \rightarrow \mathscr{A}_{m^{\prime}} \uparrow \Delta$ such that $\phi(B)$ is the Schauder basis over $\Delta$. Further, for each $i=1,2, \ldots$, as shown in the proof of Lemma 2.6. it is safe to take $m^{\prime}=m$, and to assume that the 1-dimensional cones of $\Delta$ are the rays spanned by the standard basis $\left\{e_{1}, \ldots, e_{m}\right\} \subseteq \mathbb{R}^{m}$. The set $\phi\left(B_{i}\right)$ is the Schauder basis over a unimodular fan $\Delta_{i}$ that is obtained from $\Delta$ via the finite sequence of (regular) stellar subdivisions corresponding to the one producing $B_{i}$ from $B$. To simplify notation, we can safely assume in the rest of this proof that $\phi$ is the identity.

We prove that for every integer $n \geqslant 0$ the set

$$
\bigcup_{i=0}^{n} \hat{B}_{i}=\left\{b_{1}, \ldots, b_{m}, b_{m+1}, \ldots, b_{m+n}\right\},
$$

where $\hat{B}_{i}=\left\{b_{1}, \ldots, b_{m+i}\right\}$, is linearly independent in $G$. For this, consider a linear dependence

$$
z_{1} b_{1}+\cdots+z_{m} b_{m}+z_{m+1} b_{m+1}+\cdots+z_{m+n} b_{m+n}=0
$$

where each $z_{j}$ is an integer, $j=1, \ldots, m+n$. The elements $b_{1}, \ldots, b_{m}$ form the Schauder basis over $\Delta$, and so are in bijection with the primitive (standard basis) vectors $e_{1}, \ldots, e_{m}$ in $|\Delta|$ that span the 1-dimensional cones of $\Delta$; further, $b_{j}\left(e_{j}\right)=1$, $b_{j}\left(e_{j^{\prime}}\right)=0$ if $j^{\prime} \neq j, j=1, \ldots, m$. Now observe that, by inspection of $(\hat{\dagger})$, each $b_{m+i}$ is a Schauder hat over the unimodular fan $\Delta_{i}$ obtained from $\Delta$ by $i$ stellar subdivisions. But then contemplation of the definitions of the Schauder hat and stellar subdivision shows that $b_{m+i}$ vanishes over each $e_{1}, \ldots, e_{m}$. Evaluating (D) at each $e_{j}, j=1, \ldots, m$, we thus infer

$$
z_{1}=\cdots=z_{m}=0
$$

Next consider $z_{m+1}$. Again by $(\hat{\dagger}), b_{m+1}$ is the Schauder hat (of the Schauder basis over $\Delta_{1}$ ) at the unique 1-dimensional cone $\sigma_{1}$ that lies in $\Delta_{1}$, but not in $\Delta$. Let $v_{m+1}$ be the unique primitive vector in $|\Delta|$ that spans $\sigma_{1}$. Following the notation in (耳) and $(\hat{\dagger})$, let $h^{(0)}, k^{(0)}$ denote the unique elements of $\hat{B}_{0}$ such that $b_{m+1}=h^{(0)} \wedge k^{(0)}$. Taking $\left(\mathrm{Z}_{0}\right)$ into account, we see that in (D) we must have

$$
z_{m+1}=0
$$


Indeed, by ( $\hat{\dagger})$, the only hats in $\left\{b_{1}, \ldots, b_{m}, b_{m+1}, \ldots, b_{m+n}\right\}$ that may not vanish at $v_{m+1}$ beside $b_{m+1}$ bear an index $<m+1$. Evaluating (D) at $v_{m+1}$ and using $\left(\mathrm{Z}_{0}\right)$ we thus infer $\left(Z_{1}\right)$. Iterating this argument we eventually conclude that $z_{1}=\cdots=$ $z_{m+n}=0$, as was to be shown. This proves that $\bigcup_{i=0}^{n} \hat{B}_{i}$ is linearly independent in $G$, and therefore $\hat{B}_{\infty}$ is, too.

Since it is obvious that each element of $\hat{B}_{\infty}$ is non-negative, and hence positive by the linear independence of $\hat{B}_{\infty}$, it only remains to show that every $g \in G$ is in the subgroup of $G$ generated by $\hat{B}_{\infty}$. To this aim, let us first prove by induction on $i$ that each set $B_{i}$ lies in the subgroup generated by $\hat{B}_{\infty}$. For $i=0, \hat{B}_{0}=B \subseteq \hat{B}_{\infty}$, and there is nothing to prove. Next assume $B_{i} \subseteq\left[\hat{B}_{\infty}\right]$ for some $i \geqslant 0$, with the intent of proving that $B_{i+1} \subseteq\left[\hat{B}_{\infty}\right]$, too. With the notation of (†) and $(\hat{\dagger})$ we have

$$
B_{i+1}=\left(B_{i} \backslash\left\{h^{(i)}, k^{(i)}\right\}\right) \cup\left\{h^{(i)}-\left(h^{(i)} \wedge k^{(i)}\right), k^{(i)}-\left(h^{(i)} \wedge k^{(i)}\right), h^{(i)} \wedge k^{(i)}\right\} .
$$

By construction, we also have $h^{(i)} \wedge k^{(i)} \in \hat{B}_{\infty}$. By the induction hypothesis we have $B_{i} \subseteq\left[\hat{B}_{\infty}\right]$, and since $h^{(i)}, k^{(i)} \in B_{i}$, in particular $h^{(i)}, k^{(i)} \in\left[\hat{B}_{\infty}\right]$. But then both $h^{(i)}-\left(h^{(i)} \wedge k^{(i)}\right)$ and $k^{(i)}-\left(h^{(i)} \wedge k^{(i)}\right)$ lie in $\left[\hat{B}_{\infty}\right]$, and hence $B_{i+1} \subseteq\left[\hat{B}_{\infty}\right]$, as was to be shown.

It now suffices to show that for each $g \in G$ there is an index $i_{0}$ such that $g \in\left[B_{i_{0}}\right]$, since we just proved that $\left[B_{i}\right] \subseteq\left[\hat{B}_{\infty}\right]$ for all $i=0,1, \ldots$ For this, recall our identification of $G$ with $\mathscr{A}_{m}\left\lceil\Delta\right.$, where $\Delta$ is a unimodular fan in $\mathbb{R}^{m}$ whose 1-dimensional cones are spanned by the standard basis $\left\{e_{1}, \ldots, e_{m}\right\} \subseteq \mathbb{R}^{m}$, and of $B$ with the Schauder basis over $\Delta$. Then each non-zero element $l=z_{1} b_{1}+\cdots+z_{m} b_{m} \in[B]$ uniquely determines a hyperplane in $\mathbb{R}^{m}$, namely, the hyperplane $L_{l} \subseteq \mathbb{R}^{m}$ whose unit normal vector is in direction $\left(z_{1}, \ldots, z_{m}\right)$. By (i) in this theorem, the sequence $B=B_{0}, B_{1}, \ldots, B_{n}, \ldots$ splits each element $l$; that is, there is an index $i_{0}$ such that the Schauder basis $B_{i_{0}}=\left\{h_{1}, \ldots, h_{u}\right\}$ over $\Delta_{i_{0}}$ satisfies $w_{1} h_{1}+\cdots+w_{u} h_{u}=l$ for (uniquely determined) integers $w_{j} \in \mathbb{Z}$ such that whenever $w_{j_{1}} w_{j_{2}}<0$, then $h_{j_{1}} \wedge h_{j_{2}}=0$. By a simple computation, this fact can be geometrically reformulated by saying that the set $L_{l} \cap|\Delta|$ is a union of cones in $\Delta_{i_{0}}$, or, equivalently, that each cone of $\Delta_{i_{0}}$ is contained in one of the two closed half-spaces in $\mathbb{R}^{m}$ determined by $L_{l}$. As first shown in 12, this entails that the sequence $\Delta=\Delta_{0}, \Delta_{1}, \ldots, \Delta_{n}, \ldots$ eventually refines any unimodular fan whose support is $\Delta$. Since, by a standard argument, there is a unimodular fan $\Sigma$ with support $\Delta$ such that the piecewise linear function $g$ is linear over each cone of $\Sigma$ (cf. e.g. [13, Thm. 8.5]), we conclude that there exists $i_{0}$ such that $g$ is linear over each cone of $\Delta_{i_{0}}$. But then $g$ is immediately seen to lie in the subgroup generated by $B_{i_{0}}$, the Schauder basis over $\Delta_{i_{0}}$.

The last assertion of the Theorem is now clear, recalling that simplicial groups are free Abelian groups of finite rank.

\section{ACKNOWLEDGEMENT}

The author visited the University of Cambridge for the first time in Michaelmas Term 2001, as a Ph.D. student. During that visit, thanks to his host, Andrew M.W. Glass, he began to grasp the profound connections between recursion theory and the theory of groups. He has since learned from Andrew many more things than can be mentioned here. This piece of work is dedicated to him as a token of gratitude and friendship. 


\section{REFERENCES}

[1] P. S. Alexandrov, Combinatorial topology, Dover Publications Inc., Mineola, NY, 1998, Translated from the Russian. Reprint of the 1956, 1957 and 1960 translations. MR.1643155 (99g:55001)

[2] R. Baer, Abelian groups without elements of finite order, Duke Math. J. 3 (1937), no. 1, 68-122. MR 1545974

[3] K. A. Baker, Free vector lattices, Canad. J. Math. 20 (1968), 58-66. MR0224524 (37:123)

[4] W. M. Beynon, Combinatorial aspects of piecewise linear functions, J. London Math. Soc. (2) 7 (1974), 719-727. MR0332617 (48:10943)

[5] - Duality theorems for finitely generated vector lattices, Proc. London Math. Soc. (3) 31 (1975 part 1), 114-128. MR0376480 (51:12655)

[6] Applications of duality in the theory of finitely generated lattice-ordered abelian groups, Canad. J. Math. 29 (1977), no. 2, 243-254. MR0437420 (55:10350)

[7] A. Bigard, K. Keimel, and S. Wolfenstein, Groupes et anneaux réticulés, Lecture Notes in Mathematics, Vol. 608, Springer-Verlag, Berlin, 1977. MR0552653 (58:27688)

[8] R. D. Bleier, Archimedean vector lattices generated by two elements, Proc. Amer. Math. Soc. 39 (1973), 1-9. MR0329997 (48:8336)

[9] F. Borceux, Handbook of categorical algebra. 1, Encyclopedia of Mathematics and its Applications, vol. 50, Cambridge University Press, Cambridge, 1994. MR1291599 (96g:18001a)

[10] _ Handbook of categorical algebra. 2, Encyclopedia of Mathematics and its Applications, vol. 51, Cambridge University Press, Cambridge, 1994. MR1313497 (96g:18001b)

[11] M. R. Darnel, Theory of lattice-ordered groups, Monographs and Textbooks in Pure and Applied Mathematics, vol. 187, Marcel Dekker Inc., New York, 1995. MR.1304052 (95k:06032)

[12] C. De Concini and C. Procesi, Complete symmetric varieties. II. Intersection theory, Algebraic groups and related topics (Kyoto/Nagoya, 1983), Adv. Stud. Pure Math., vol. 6, North-Holland, Amsterdam, 1985, pp. 481-513. MR.803344 (87a:14038)

[13] G. Ewald, Combinatorial convexity and algebraic geometry, Graduate Texts in Mathematics, vol. 168, Springer-Verlag, New York, 1996. MR.1418400 (97i:52012)

[14] A. M. W. Glass, Partially ordered groups, Series in Algebra, vol. 7, World Scientific Publishing Co. Inc., River Edge, NJ, 1999. MR1791008 (2001g:06002)

[15] A. M. W. Glass and J. J. Madden, The word problem versus the isomorphism problem, J. London Math. Soc. (2) 30 (1984), no. 1, 53-61. MR760872 (86i:03059)

[16] A. M. W. Glass and V. Marra, The underlying group of any finitely generated abelian lattice-ordered group is free, Algebra Universalis 56 (2007), no. 3-4, 467-468. MR2318248 (2008b:06015)

[17] A. Hatcher, Algebraic topology, Cambridge University Press, Cambridge, 2002. MR 1867354 (2002k:55001)

[18] J. M. Lee, Introduction to topological manifolds, Graduate Texts in Mathematics, vol. 202, Springer-Verlag, New York, 2000. MR1759845 (2001d:57001)

[19] J. J. Madden, Two methods in the study of k-vector lattices, Ph.D. thesis, Wesleyan University, 1983. MR2633496

[20] C. Manara, V. Marra, and D. Mundici, Lattice-ordered abelian groups and Schauder bases of unimodular fans, Trans. Amer. Math. Soc. 359 (2007), no. 4, 1593-1604. MR2272142 (2007m:06022)

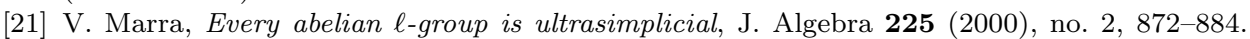
MR.1741567 (2001c:06020)

[22] Weinberg's theorem, Elliott's ultrasimplicial property, and a characterisation of free lattice-ordered Abelian groups, Forum Math. 20 (2008), no. 3, 505-513. MR2418203 (2009b:06037)

[23] E. E. Moise, Geometric topology in dimensions 2 and 3, Graduate Texts in Mathematics, Vol. 47, Springer-Verlag, New York, 1977. MR0488059 (58:7631)

[24] D. Mundici, Farey stellar subdivisions, ultrasimplicial groups, and $K_{0}$ of $A F C^{*}$-algebras, Adv. in Math. 68 (1988), no. 1, 23-39. MR.931170 (89d:46072)

[25] D. Mundici, Free generating sets of lattice-ordered abelian groups, J. Pure Appl. Algebra 211 (2007), no. 2, 400-403. MR2340457 (2008k:06021)

[26] _ Revisiting the free 2-generator abelian l-group, J. Pure Appl. Algebra 208 (2007), no. 2, 549-554. MR2277694 (2007k:06024) 
[27] T. Oda, Convex bodies and algebraic geometry, Ergebnisse der Mathematik und ihrer Grenzgebiete (3), vol. 15, Springer-Verlag, Berlin, 1988. MR922894 (88m:14038)

[28] C. P. Rourke and B. J. Sanderson, Introduction to piecewise-linear topology, Springer-Verlag, Berlin, 1982. MR665919 (83g:57009)

[29] Z. Semadeni, Schauder bases in Banach spaces of continuous functions, Lecture Notes in Mathematics, vol. 918, Springer-Verlag, Berlin, 1982. MR653986 (83g:46023)

Dipartimento di Informatica e Comunicazione, Università degli Studi di Milano, via Comelico 39/41, I-20135 Milano, Italy

E-mail address: vincenzo.marra@unimi.it

Current address: Dipartimento di Matematica "Federigo Enriques", Università degli Studi di Milano, via Cesare Saldini 50, I-20133 Milano, Italy 